\title{
Modeling and sensitivity analyses of VSP based virtual inertia controller in HVDC links of interconnected power systems
}

\author{
Elyas Rakhshani $^{\mathrm{a}, \mathrm{b}, *}$, Daniel Remon ${ }^{\mathrm{b}}$, A.M. Cantarellas ${ }^{\mathrm{a}, \mathrm{b}}$, Jorge M. Garcia ${ }^{\mathrm{a}}$, \\ Pedro Rodriguez ${ }^{a, b}$ \\ a Abengoa, Campus Palmas Altas, 41014 Seville, Spain \\ b Technical University of Catalonia (UPC), Electrical Engineering Department, Barcelona, Spain
}

\section{A R T I C L E I N F O}

Keywords:

Active power control

Automatic generation control

HVDC interconnected systems

Virtual inertia

\begin{abstract}
A B S T R A C T
Virtual inertia is known as an inevitable part of the modern power systems, especially considering the increasing level of power electronic-based component and HVDC interconnection in the future gird. To cope with this challenging issue, a novel approach for modeling and analysis the effect of virtual inertia on frequency stability of multi area interconnected system is proposed. The proposed method is based on Virtual Synchronous Power (VSP) control strategy of HVDC interconnected systems. The presented work is focused on modeling and detailed sensitivity analysis of VSP-based HVDC concept in frequency control and automatic generation control task of interconnected power systems. In order to understand the influence of virtual inertia parameters, a set of sensitivity analysis is performed to identify the proper range of critical control parameters on the stability of the system. The effectiveness of the proposed concept on dynamic improvements is also validated through Matlab simulation of multiarea (two-area and three-area) test systems. The three-area test system is based on complex model of 39-bus, 10 generator test system.
\end{abstract}

\section{Introduction}

One of the main concerns in an electrical power system is to ensure the balance between the total power generation with the total load demand and, then regulating the system frequency and tie-line power exchange [1,2]. To supply a high quality and reliable electric power to consumers, the frequency control will be one of the most profitable ancillary services in power system design and operation [3]. This task in the presence of renewable generation and complex HVDC interconnected systems will become challenging issue [4].

In the modern scenario of power system, there is an increasing attention to expansion of interconnected power grid using HVDC transmission lines. This is due to several limitations associated with AC lines, especially for long distance connections. HVDC interconnection is one of the main applications of power converters in multi-area interconnected power systems which could bring several advantages, e.g., fast and bidirectional controllabil-

\footnotetext{
* Corresponding author at: Technical University of Catalonia (UPC), Electrical Engineering Department, SEER Research Center, GAIA building, 22, Rambla Sant Nebridi, 08222 Terrassa, Barcelona, Spain.

E-mail address: elyas.rakhshani@gmail.com (E. Rakhshani).
}

ity, damping capabilities and frequency stability support [5-7]. HVDC systems can essentially improve the reliability of complex interconnected systems. It can act as a kind of firewall against cascading disturbances to prevent global blackouts [8,9]. It is worth to mention that, the HVDC links are superior to the governor which is a conventional frequency control system in terms of highspeed performance. With a proper coordination and control of the HVDC link, the tie-line power modulation of the HVDC link through interconnections is applicable for stabilizing the frequency oscillations of AC systems by adding more damping to the system [10]. Typically, conventional generators with the droop capability are responsible for providing sufficient inertia against frequency deviations in the system. Nowadays, considering the high penetration of renewable generations and increasing application of power electronic-based components, the system's inertia is becoming low. Therefore, the lack of sufficient inertia will be the main concern of future grids $[10,11]$. Therefore, control of frequency and tie-line power exchange between authorized areas will be more and more complex. In such scenario, the matter of modeling and control considering the methods of providing virtual inertia to the system is critical and the role of advanced technologies such as the use of modern power processing systems, energy storage, and advanced converters in HVDC links will be essential. 
Inertia emulation based on the derivative of frequency is one of the common techniques for emulating inertia [12]. Derivative of grid frequency can be used as a complementary signal for VSC controllers which is supported by proper communication channels [13]. Inertia emulation controller (INEC) is another method implemented for providing inertia by a DC link in Multi-Terminal HVDC systems [14]. This controller is based on the error signal coming from frequency deviation measurement from the AC grid, generating the DC voltage reference for emulating transient inertia using DC capacitance of the DC grid. The method is simple and generic with some limitation for real application of the power industry. In general, virtual emulation based on derivative term of frequency is very are always dependent on proper estimation of frequency which can bring some limitations. In most of these methods, the use of Phase Locked Loop (PLL) is essential for a proper estimation and measurements of the signals. Any amplified noise caused by frequency deviation measurements could bring instability problems. This limitation can be even worse, especially during abnormal faults and unsymmetrical system. The synchronization system used to generate errors in the detection of the grid voltage magnitude and phase-angle at the point of connection. In such scenario, in which a fast response from the generator is required, the errors made by the synchronization system give rise to uncontrolled power exchanges with the grid. Therefore, better methods of providing alternative inertia without limitation of signal measurements are necessary; which is the topic that is going to be proposed in this paper.

The present paper addresses a new LFC scheme considering the high level control application of the concept of Virtual Synchronous Power (VSP) through the converter stations of the HVDC link for inertia emulation in multi-area systems. The used VSP is based on the synchronous controller, which was reported in 2012 [15]. This control strategy is proposing a new method in power converter control behaving as a synchronous generator with the ability of emulating synchronous inertia without the drawbacks of conventional generators [16].

The main objective of this paper is to propose a new approach of frequency stability analysis in multi-area AGC system, adding the VSP concept in HVDC links of interconnected systems. In the last several decades, traditional Load Frequency Control (LFC) models have been modified and revised to add different functionalities in the reformulation of conventional power systems. Most of those modifications are related to AGC in a deregulated market scenario [17], different types of power plants like renewable generation [18], the demand side dynamic models [19] and recently implementing the effects of PLL and frequency measurements [20]. The importance of the VSP concept in providing ancillary services like emulating simultaneous damping and inertia for frequency control improvements is another motivation of this paper. Another goal of this paper is to propose a model which is very useful for pre-evaluation of the dynamic effects of converter stations of the HVDC link in higher level control design for power systems applications. Proper range of control parameters of the VSP is explored through detailed sensitivity analysis. This analysis can be used as a basic methodology for analyzing the effects of control gains on the system performance.

\section{Dynamic model of multi-area AGC system}

\subsection{Conventional frequency regulation}

A proper control strategy for active power/frequency issue in large scale power systems is shown in Fig. 1. A low-order linearized model could be used for modeling the load-generation dynamic behaviors. The frequency deviation for $i$ th area could be as follows:

$\Delta \omega_{i}=\frac{K_{p i}}{1+s T_{p i}}\left[\Delta P_{m i}-\Delta P_{L i}-\Delta P_{\text {tie }, i}\right]$

$\Delta P_{m i}=\sum_{j=1}^{l} \Delta P_{m i j}$

where $K_{p i}$ and $T_{p i}$ are gain and time constant of power system and can be calculated considering the constant of inertia $(M=2 H)$ and damping $(D)$ of the power system area $\left(T_{p}=2 H / D\right.$ and $\left.K_{p}=1 / D\right)$. Moreover, $\Delta P_{m i}$ in Eq. (1) is the total generated power by GENCOs (Generation Companies) and $\Delta P_{L}$ is load change in each area. While $\Delta P_{m i j}$ is the output of each unit:

$\Delta P_{m i j}=\frac{1}{1+s T_{t g, i j}}\left[\frac{-\Delta \omega_{i}}{R_{i}}-K_{I} a p f_{i j} \Delta P_{\text {refi }}\right]$

$\Delta P_{\text {refi }}=\frac{\mathrm{ACE}_{i}}{S}$

where $T_{t g, i j}$ is the time constant of each turbine-governor unit, $R_{i}$ is the droop characteristic of equivalent generation unit, $K_{I}$ is the integral controller gain, $\Delta P_{\text {refi } i}$ is the area reference power which is the integration of ACE. The ACE is the area control error expressed as a linear combination of tie-line power flow and weighted frequency deviations.

$\mathrm{ACE}_{i}=\beta_{i} \Delta \omega_{i}+\Delta P_{\text {tie }, i}$

where $\beta$ is the bias coefficient. The participation of individual generation output for each unit in AGC will be accomplished by area participation factors (apf) in each area.

For modeling the interconnections between $N$ areas in multiarea AGC system, the tie-line power exchange between area $i$ and the rest of area could be presented as follows [7]:

$\Delta P_{\text {tie }, i}=\sum_{\substack{j=1 \\ j \neq i}}^{N} \Delta P_{\text {tie }, i j}=\frac{1}{s}\left[\sum_{\substack{j=1 \\ j \neq i}}^{N} T_{i j} \Delta \omega_{i}-\sum_{j=1}^{N} T_{i j} \Delta \omega_{j}\right]$

$\Delta P_{\text {tie }, j i}=-\Delta P_{\text {tie }, i j}$

where $T_{i j}$ is the synchronizing coefficient between areas. Therefore, considering $E_{i}$ and $\delta_{i 0}$ as the initial values of voltage and angle of $i$ th terminal and $X$ as the reactance of the line, we have:

$T_{i j}=\frac{E_{1} E_{2}}{X} \cos \left(\delta_{10}-\delta_{20}\right)$

\section{2. $A C / D C$ transmission model}

In case of small signal modeling and stability studies for the pre-design process of higher level control design like AGC, each converter station could be modelled as a first-order transfer function consisting a proper time constant. For HVDC system with two VSC stations, the second-order transfer function will be approximated by equivalent first-order transfer function imitating the overall time response of HVDC system as follow:

$\frac{1}{1+s T_{1}} \times \frac{1}{1+s T_{2}}=\frac{1}{1+\left(T_{1}+T_{2}\right) s+\left(T_{1} T_{2}\right) s^{2}} \cong \frac{1}{1+s T_{D C}}$

where $T_{1}$ and $T_{2}$ are the time constant of converters and $T_{\mathrm{DC}}$ is the equivalent time constant of overall HVDC control system:

$T_{\mathrm{DC}}=T_{1}+T_{2}$ 


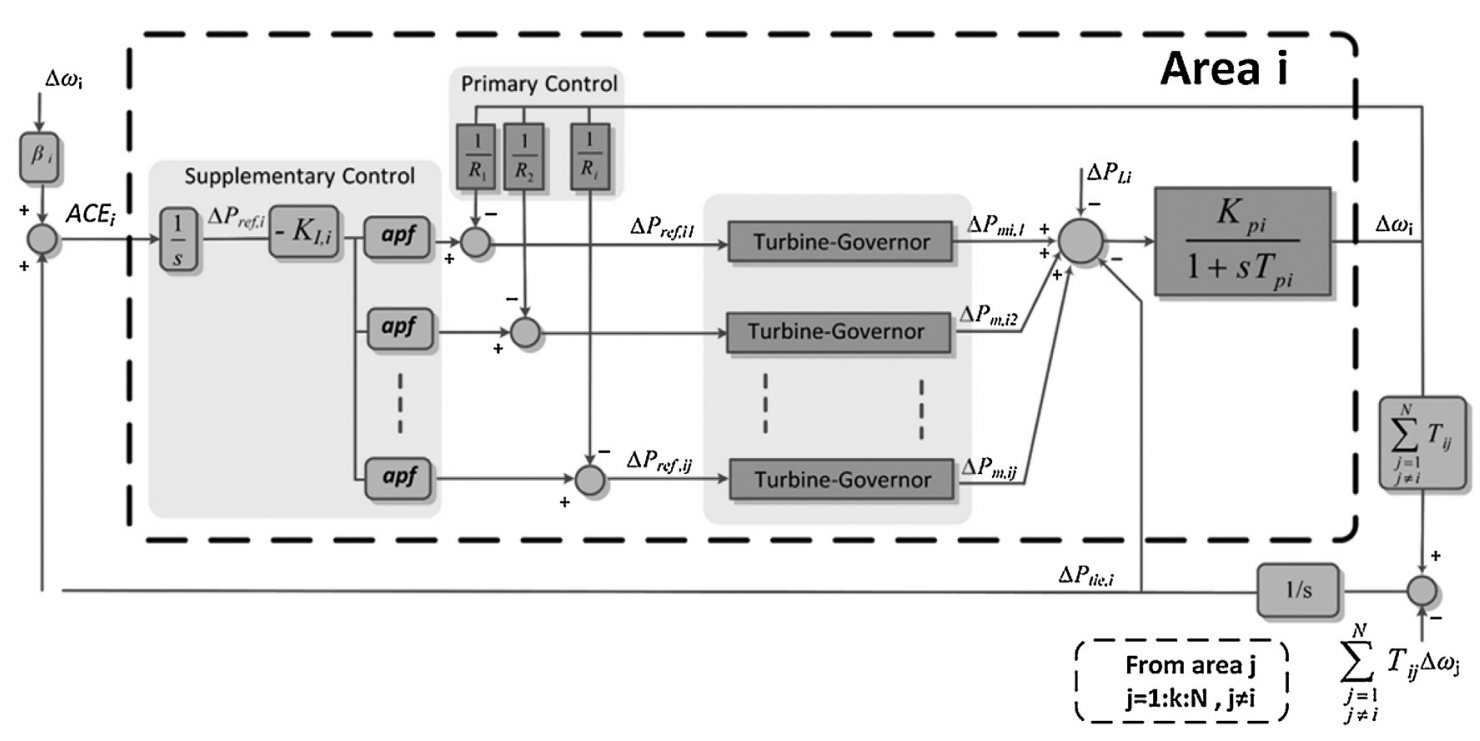

Fig. 1. Basic frame of $i$ th area in AGC implementation of multi-area interconnected power system.

Therefore, the incremental power flow through the HVDC transmission system could be modelled by a linear first-order model with a proper time constant as follows:

$T_{\mathrm{DC}} \frac{\mathrm{d} \Delta P_{\text {tie, } \mathrm{DC}}}{\mathrm{d} t}=\Delta P_{\text {ref, } \mathrm{DC}}-\Delta P_{\text {tie, } \mathrm{DC}}$

where $\Delta P_{\text {ref,DC }}$ is the reference control signal of DC power and $\Delta P_{\text {tie, DC }}$ will be the real DC power flow through the system. It is assumed that the DC line is lossless and DC power flow out from one converter to another converter. As shown in Fig. 2, there is a DC line in parallel coordination with AC line between Area $i$ and Area $k$.

The $\Delta P_{\text {ref,DC }}$ is the input signal of the HVDC system which will be generated by different control signals. These signals are frequency deviations of each interconnected areas and AC power flow deviations. This power modulation controller is modeled as a proportional controller.

$\Delta P_{\text {ref, DC }}=K_{f i} \Delta \omega_{i}+K_{f k} \Delta \omega_{k}+K_{\mathrm{AC}} \Delta P_{\text {tie }, i k}$

Considering this new state in DC link, the new ACE signal of each area in AGC operation which contains some additional HVDC link will be adapted:

$\Delta P_{\text {tot }, i}=\Delta P_{\text {tie }, \mathrm{DC}}+\Delta P_{\text {tie }, i}$

$\mathrm{ACE}_{\text {new }, i}=\beta_{i} \Delta \omega_{i}+\Delta P_{\text {tot }, i}$

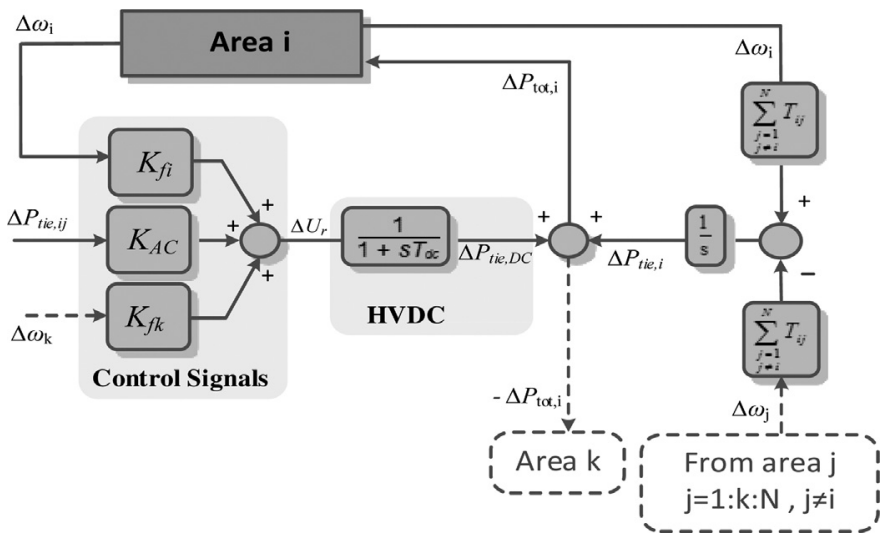

Fig. 2. Control actions of interconnected area with HVDC model. where $\Delta P_{\text {tot }, i}$ is the total tie-line power deviations, $\Delta P_{\text {tie }, i}$ is the tieline power exchange between Area $i$ and other areas and $\Delta P_{\text {tie, DC }}$ is the DC power deviation in the HVDC link between Area $i$ and Area $k$. In this study, the sign convention for net power into a control area is negative, and net power out of a control area is positive. It should be mentioned that the role of ACE signal in LFC is to maintain the frequency and power interchanges at the scheduled values. ACE signal will represent the real power imbalance between generation and load. In practice, ACE will be performed by its supervisory control considering the proportional integral controller, limiters and delays. As explained before, this signal will be distributed among LFC participant generation units with their participation factors for providing proper control command for set-points of specified plants.

\section{Virtual synchronous power strategy}

In this section the concept of Virtual Synchronous Power (VSP) which is based on the control of voltage source converter through an active power synchronization loop and a virtual admittance is presented. The general structure of synchronous power control for each converter of the VSC-HVDC line is presented in Fig. 3. This control structure stems from programming the electrical performance of a Synchronous Generator (SG) in a digital frame-work which is responsible for controlling the VSC-converter. However, it is worth to point that this synchronous controller does not aim to faithfully mimic the response of the synchronous generator, but it overcomes the drawbacks of the inherent oscillatory response of the conventional synchronous generator [16]. It should be note that, in this scheme the protection controllers are not included. The DC energy storage element represented by $C$ in Fig. 3 , is in charge of absorption of any transient unbalance between the two power converters connected to the DC link. This energy storage element can be inserted in the converter topology or implemented by any additional power converter storage.

By modifying the swing equation of conventional synchronous generator, the general electromechanical control loop for VSP application in transient contingencies can be presented based on the diagram of Fig. 4. This diagram represents a Power-Locked Loop, in which any variation between the delivered power by the converter $\left(P_{\text {out }}\right)$ and the input power from the DC side, $\left(P_{\text {in }}\right)$ is processed by a Power Loop Controller (PLC) to set a relative frequency that should be added to the synchronous frequency of 


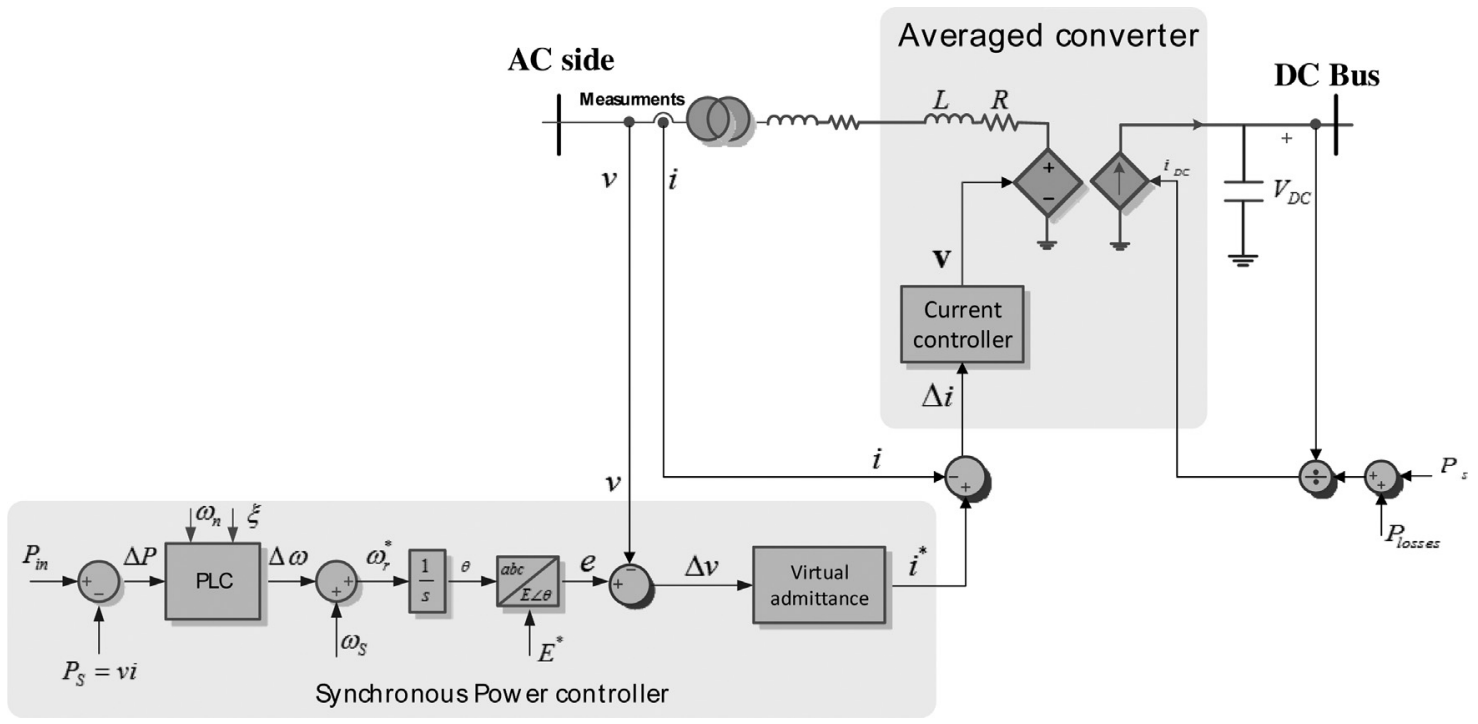

Fig. 3. The configuration of simplified synchronous power controller.

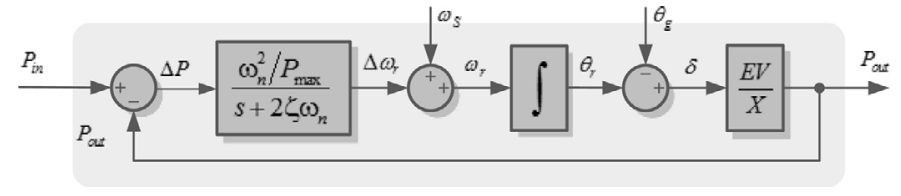

Fig. 4. Active power loop representation of the overall VSP controller.

the grid, for generating the rotating frequency of a virtual rotor. The integral of such frequency gives the angular position of the virtual rotor leading to the power delivered by the power converter.

The transfer function of a simple controller for PLC which will represent the mechanical behavior of synchronous generator might be written as:

$\operatorname{PLC}(s)=\frac{N(s)}{D(s)}=\frac{\omega_{n}^{2} / P_{\max }}{s+2 \zeta \omega_{n}}$

while $\zeta$ is damping factor, $\omega_{n}$ is the natural frequency and $P_{\max }$ is the maximum active power that can be delivered:

$P_{\max }=\frac{E V}{X}$

where $E, V$ and $X$ are internal emf voltage, grid voltage and overall impedance, respectively. Usually, the values for $E$ and $V$ are close to the rated ones and the value for the load angle $(\delta)$, used to be very small. Under such conditions, the value of $\sin (\delta)$ can be simply approximated by $\delta$, and thereby the delivered electrical power can be approximated to $(P=E V / X \delta)$. The transmitted power could be easily adjusted by shifting the output voltage phase of the converter forward or backward. It should be noted that the value of $P_{\max }$ will determine the range of the power for the installed converter. A straightforward analysis of such electromechanical model leads to the following transfer function describes the dynamic relationship between the input and output powers [15]:

$\frac{P_{\text {out }}}{P_{\text {in }}}=\frac{\omega_{n}^{2}}{s^{2}+2 \zeta \omega_{n} s+\omega_{n}^{2}}=\frac{P_{\max } / J \times \omega_{s}}{s^{2}+k / J \times \omega_{s} s+P_{\max } / J \times \omega_{s}}$

where $P_{\text {in }}, P_{\text {out }}, J, k$ and $\omega_{s}$ are input power, delivered active power, the moment of inertia, damping constant and synchronous frequency. From this transfer function, the relationships of natural frequency, $\omega_{n}$, and the damping factor, $\zeta$, of the virtual electromechanical system to the rest of parameters would be given by:

$\omega_{n}=\sqrt{\frac{P_{\max }}{J \times \omega_{s}}}$

$\zeta=\frac{k}{2 \sqrt{P_{\max } J \times \omega_{s}}}$

Therefore, the virtual inertia $(J)$ and desired damping to the system could be emulated by proper selections of these control parameters of VSP strategy. In practice, synchronous generators are equipped with some damping windings to attenuate these power oscillations in a certain extent. However, the damping action provided by such winding is limited by some mechanical and electrical constraints, thereby the conventional synchronous generators do not have a perfectly damped performance. Due to the fact that a VSP-based control behaves as a programmable synchronous generator, it is possible to adjust and program its capability for damping power oscillations in the power system with an optimal performance.

\section{The proposed approach with VSP-based HVDC}

As explained in the previous section, the dynamic relationships between input and output power of each converter station could be a second-order transfer function. The relationship between input and output signals in this second-order system could be identified using its characteristic equation of the VSP dynamic model:

$\ddot{y}+2 \zeta \omega_{n} \dot{y}+\omega_{n}^{2} y=\omega_{n}^{2} u$

The input signal, $u$, is the reference for DC power emulation which is related to other state of the global multi-area system and the output signal will consist of two state variables.

$\Delta Y_{\mathrm{VSP}}=\left[\begin{array}{c}\Delta X_{1, \mathrm{VSP}} \\ \Delta X_{2, \mathrm{VSP}}\end{array}\right]$

where $\Delta X_{1, \mathrm{VSP}}$ represents the emulated power ( $\left.\Delta P_{\mathrm{DC}, \mathrm{VSP}}\right)$ and $\Delta X_{2, \mathrm{VSP}}$ is the derivative term of this power for each VSP, 


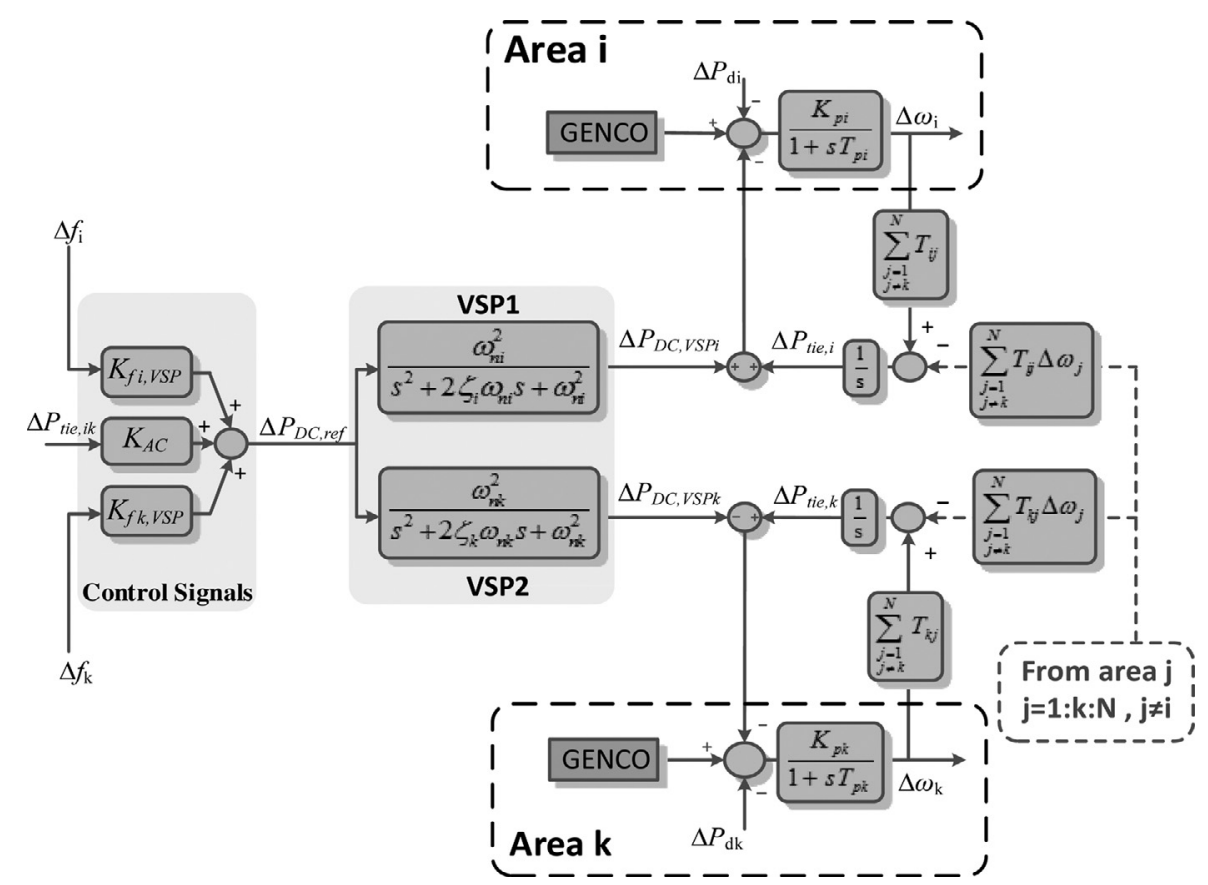

Fig. 5. Basic frame of AGC implementation in multi-area interconnected systems with a VSP based AC/DC transmission.

respectively. Based on classic control concepts, this second-order system could be represented by a set of two linear state equations.

$$
\left[\begin{array}{c}
\Delta \dot{X}_{1, \mathrm{VSP}} \\
\Delta \dot{X}_{2, \mathrm{VSP}}
\end{array}\right]=\left[\begin{array}{cc}
0 & 1 \\
-\omega_{n}^{2} & -2 \zeta \omega_{n}
\end{array}\right]\left[\begin{array}{c}
\Delta X_{1, \mathrm{VSP}} \\
\Delta X_{2, \mathrm{VSP}}
\end{array}\right]+\left[\begin{array}{c}
0 \\
\omega_{n}^{2}
\end{array}\right] \Delta U
$$

where $U$ is the reference signal coming from higher level control which is defined as:

$$
\Delta U=\Delta P_{\mathrm{DC}, \mathrm{ref}}=K_{f i, \mathrm{VSP}} \Delta \omega_{i}+K_{f k, \mathrm{VSP}} \Delta \omega_{k}+K_{\mathrm{AC}} \Delta P_{\text {tie }, i k}
$$

As the HVDC line is located between Area $i$ and Area $k$, the frequency deviation of those areas is the most suitable control signal. This state space presentation will be part of the global system and it could be added to the rest of the state space model.

As shown in Fig. 5, it is assumed that there is a parallel AC/HVDC link between Area $i$ and Area $k$, where both converter stations of HVDC link are facilitated by VSP functionalities.

The dynamic equations of the AC/DC interconnected areas considering the dynamic of VSP based HVDC link in Laplace domain will be as follows:

$$
\begin{aligned}
& \Delta \omega_{i}=\frac{K_{p i}}{1+s T_{p i}}\left[\Delta P_{m i}-\Delta P_{L 1}-\left(\Delta P_{\mathrm{tie}, i}+\Delta X_{1, \mathrm{VSP} i}\right)\right] \\
& \Delta \omega_{k}=\frac{K_{p k}}{1+s T_{p k}}\left[\Delta P_{m k}-\Delta P_{L k}-\left(\Delta P_{\mathrm{tie}, k}+\Delta X_{1, \mathrm{VSP} k}\right)\right] \\
& \mathrm{ACE}_{i}=\beta_{i} \Delta \omega_{i}+\left[\Delta P_{\mathrm{tie}, i}+\Delta X_{1, \mathrm{VSP} i}\right] \\
& \mathrm{ACE}_{k}=\beta_{k} \Delta \omega_{k}+\left[\Delta P_{\mathrm{tie}, k}+\Delta X_{1, \mathrm{VSP} k}\right] \\
& \Delta P_{\mathrm{tie}, k}=-\Delta P_{\mathrm{tie}, i} \\
& s \Delta X_{1, \mathrm{VSP} i}=\Delta X_{2, \mathrm{VSP} i} \\
& s \Delta X_{2, \mathrm{VSP} i}=\left[\frac{K_{f i, \mathrm{VSP}} \omega_{n i}^{2}}{2 \pi}\right] \Delta \omega_{i}+\left[\frac{K_{f k, \mathrm{VSP}} \omega_{n i}^{2}}{2 \pi}\right] \Delta \omega_{k} \\
& \quad+\left[K_{\mathrm{AC}, \mathrm{VSP}} \omega_{n i}^{2}\right] \Delta P_{\mathrm{tie}, i k}-\omega_{n i}^{2} \Delta X_{1, \mathrm{VSP} i}-2 \zeta_{i} \omega_{n i} \Delta X_{2, \mathrm{VSP} i}
\end{aligned}
$$

$$
s \Delta X_{1, \mathrm{VSP} k}=\Delta X_{2, \mathrm{VSP} k}
$$

$$
\begin{aligned}
s \Delta X_{2, \mathrm{VSP} k}= & {\left[\frac{K_{f i, \mathrm{VSP}} \omega_{n k}^{2}}{2 \pi}\right] \Delta \omega_{i}+\left[\frac{K_{f k, \mathrm{VSP}} \omega_{n k}^{2}}{2 \pi}\right] \Delta \omega_{k} } \\
& +\left[K_{\mathrm{AC}, \mathrm{VSP}} \omega_{n k}^{2}\right] \Delta P_{\mathrm{tie}, i k}-\omega_{n k}^{2} \Delta X_{1, \mathrm{VSP} k} \\
& -2 \zeta_{k} \omega_{n k} \Delta X_{2, \mathrm{VSP} k}
\end{aligned}
$$

It should be mentioned that, the dynamic equations of other $\mathrm{AC}$ areas which are connected with AC lines will be the same as the ones presented in Section 2. The proposed formulation is a generalized form of parallel AC/DC system or any coordinated AC and DC line which are connected to the same areas.

The AC tie-line power deviation could be used as a control signal in order to achieve suitable coordination between two lines. The coordinated AC line can be a parallel or any close line to the DC link. In the case just with one DC link without a parallel AC line, this feedback signal will be zero and the rest of the models for the VSP strategy will be the same.

Therefore, in two-area AC/DC interconnected power system which will have two synchronous controllers, four new state variables of synchronous controllers will be added to the system. Therefore, the overall state space equations, used for analysis of tow are case study, will be as follows:

$A=\left[\begin{array}{l}A_{11} \\ A_{21}\end{array}\right]_{(13 \times 13)}$ 
Therefore, each sub-matrix will be as follows:

$A_{11}=\left[\begin{array}{lllllllllllll}\frac{-1}{T_{p 1}} & 0 & \frac{K_{p 1}}{T_{p 1}} & \frac{K_{p 1}}{T_{p 1}} & 0 & 0 & 0 & 0 & \frac{-K_{p 1}}{T_{p 1}} & \frac{-K_{p 1}}{T_{p 1}} & 0 & 0 & 0 \\ 0 & \frac{-1}{T_{p 2}} & 0 & 0 & \frac{K_{p 2}}{T_{p 2}} & \frac{K_{p 2}}{T_{p 2}} & 0 & 0 & \frac{K_{p 2}}{T_{p 2}} & 0 & 0 & \frac{K_{p 2}}{T_{p 2}} & 0 \\ \frac{-1}{2 \pi R_{1} T_{\operatorname{tg} 1}} & 0 & \frac{-1}{T_{\lg 1}} & 0 & 0 & 0 & \frac{-K_{I 1}}{T_{\lg 1}} & 0 & 0 & 0 & 0 & 0 & 0 \\ \frac{-1}{2 \pi R_{2} T_{\operatorname{tg} 2}} & 0 & 0 & \frac{-1}{T_{\lg 2}} & 0 & 0 & \frac{-K_{I 1}}{T_{\operatorname{tg} 2}} & 0 & 0 & 0 & 0 & 0 & 0 \\ 0 & \frac{-1}{2 \pi R_{3} T_{\operatorname{tg} 3}} & 0 & 0 & \frac{-1}{T_{\operatorname{tg} 3}} & 0 & 0 & \frac{-K_{I 2}}{T_{\operatorname{tg} 3}} & 0 & 0 & 0 & 0 & 0 \\ 0 & \frac{-1}{2 \pi R_{4} T_{\operatorname{tg} 4}} & 0 & 0 & 0 & \frac{-1}{T_{\lg 4}} & 0 & \frac{-K_{I 2}}{T_{\operatorname{tg} 4}} & 0 & 0 & 0 & 0 & 0 \\ \frac{\beta_{1}}{2 \pi} & 0 & 0 & 0 & 0 & 0 & 0 & 0 & 1 & 1 & 0 & 0 & 0 \\ 0 & \frac{\beta_{2}}{2 \pi} & 0 & 0 & 0 & 0 & 0 & 0 & -1 & 0 & 0 & -1 & 0 \\ \frac{T_{12}}{2 \pi} & -\frac{T_{12}}{2 \pi} & 0 & 0 & 0 & 0 & 0 & 0 & 0 & 0 & 0 & 0 & 0\end{array}\right]_{(9 \times 13)}$

and

$A_{21}=\left[\begin{array}{ccccccccccccc}0 & 0 & 0 & 0 & 0 & 0 & 0 & 0 & 0 & 0 & 1 & 0 & 0 \\ a_{11,1} & a_{11,2} & 0 & 0 & 0 & 0 & 0 & 0 & a_{11,9} & a_{11,10} & a_{11,11} & 0 & 0 \\ 0 & 0 & 0 & 0 & 0 & 0 & 0 & 0 & 0 & 0 & 0 & 0 & 1 \\ a_{13,1} & a_{13,2} & 0 & 0 & 0 & 0 & 0 & 0 & a_{13,9} & 0 & 0 & a_{13,12} & a_{13,13}\end{array}\right]_{(4 \times 13)}$

where

$a_{11,1}=\frac{K_{f 1, \mathrm{VSP}} \omega_{n 1}^{2}}{2 \pi}, a_{11,2}=\frac{K_{f 2, \mathrm{VSP}} \omega_{n 2}^{2}}{2 \pi}, a_{11,9}=K_{\mathrm{AC}, \mathrm{VSP}} \omega_{n 1}^{2}, a_{11,10}=-\omega_{n 1}^{2}, a_{11,11}=-2 \zeta_{1} \omega_{n 1}$

$a_{13,1}=\frac{K_{f 1, \mathrm{VSP}} \omega_{n 2}^{2}}{2 \pi}, a_{13,2}=\frac{K_{f 2, \mathrm{VSP}} \omega_{n 2}^{2}}{2 \pi}, a_{13,9}=K_{\mathrm{AC}, \mathrm{VSP}} \omega_{n 2}^{2}, a_{13,12}=-\omega_{n 2}^{2}, a_{13,13}=-2 \zeta_{2} \omega_{n 2}$

The overall system will have thirteen state variables as follows:

$x=\left[\Delta \omega_{1} \Delta \omega_{2} \Delta P_{m 1} \Delta P_{m 2} \Delta P_{m 3} \Delta P_{m 4} \Delta \mathrm{ACE}_{1} \Delta \mathrm{ACE}_{2} \Delta P_{\text {tieAC, } 12} \Delta x_{1, \mathrm{VSP} 1} \Delta x_{2, \mathrm{VSP} 1} \Delta x_{1, \mathrm{VSP} 2} \Delta x_{2, \mathrm{VSP} 2}\right]^{T}$

and control inputs are load changes in each area:

$u=\left[\Delta P_{L 1} \Delta P_{L 2}\right]^{T}$

As shown in this model, we will have more control gains $\left(\zeta_{i}\right.$ and $\omega_{n i}$ ) related to VSP controller in each station of HVDC link. Usually, it would be possible to define a cost function in two-area AGC system for obtaining the optimum values for all of these gains. These gains could be defined based on optimization theory by minimizing the following common cost function [1-5]:

$J=\int\left[\mathrm{ACE}_{1}^{2}+\mathrm{ACE}_{2}^{2}\right] \mathrm{d} t$

This cost function is the regular function which is based on the (Integral of Squared Error) ISE method [5]. It should be noted that the ACE is the area error which is consisted of frequency and tieline power deviations in each area. These control gains $\left(\zeta_{i}\right.$ and $\left.\omega_{n i}\right)$ could be obtained using the optimization toolboxes from MATLAB. The FMINCON (find minimum of constrained function) solver can be used as a classical optimization which implements the (Sequential Quadratic Programming) SQP and the interior-point method for optimization of LFC problem. For doing the optimization process depend on the system requirement, available stored energy and control limitation design, various constraints can be added during minimization of the objection function.

\section{System studies}

In order to evaluate and analyze the effects of proposed VSPbased AC/DC system on AGC dynamics and to show how the proposed approach can help the system dynamic during contingencies, a common scenario for multi-area test system is considered. All the analysis and simulations are performed in Matlab platform. It should be mentioned that the main objective of this section it to introduce the modeling and dynamic effects of VSP concept on multi-area AGC system. The general frame of two-area interconnected system with parallel AC/DC lines is depicted in Fig. 6. It is assumed that in each area two generation company (GENCO) is located.

The presented model in Fig. 6 which has HVDC link in parallel with AC line is used for simulation. It is assumed that one contingency happened as a load change in Area 1 by increasing to 0.03 p.u. at $t=3.5 \mathrm{~s}$ in Area1. System parameters considering the time constants of turbine $\left(T_{t}\right)$ and generators $\left(T_{g}\right)$ of turbine-governor units are presented in Tables 4.1 and 4.2 [2,11]. All generators participate in LFC defined by the following apfs:

$a p f_{1}=0.50, a p f_{2}=1-a p f_{1}=0.50$

$a p f_{3}=0.50, a p f_{4}=1-a p f_{3}=0.50$ 


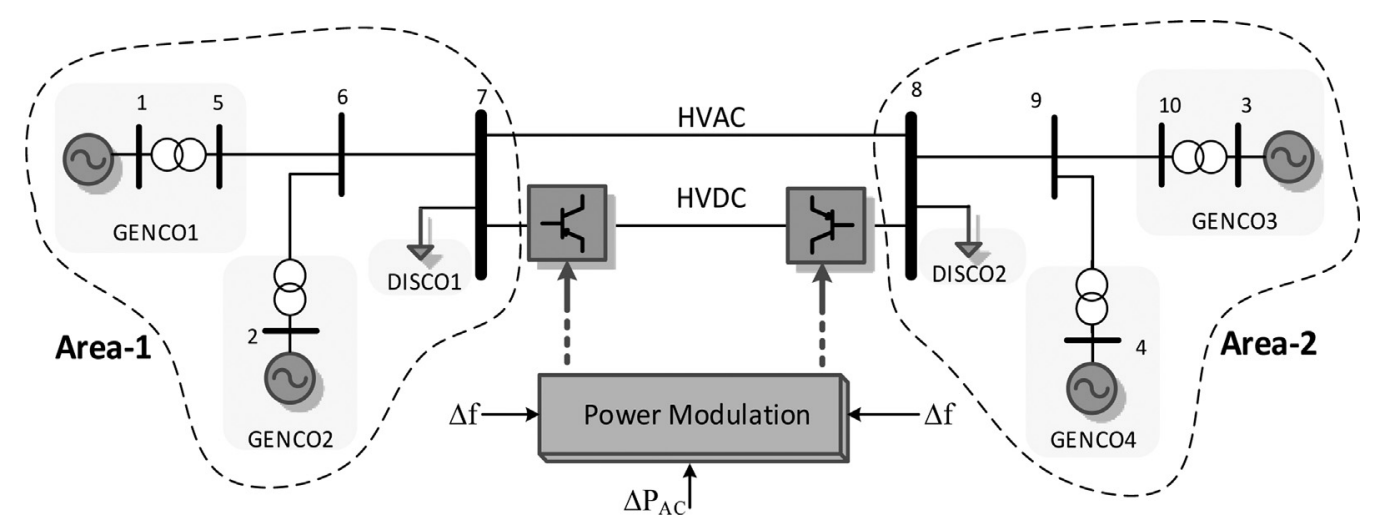

Fig. 6. The configuration of the two-area power system with HVDC link.

In this section, the dynamic effects of inertia emulation using virtual synchronous power concept on two-area AGC interconnected system are analysed by performing the numerical analysis and eigenvalue (modal) analysis. As explained before, the studied two-area power system contains of two generation units and one load demand center in each area (Fig. 6). The parameters of the studied two-area power system are given in Tables 1 and 2 and the control parameter for this case study is presented in Table 3. These values are obtained using the Eq. (33) and classical FMINCON function in Matlab software. The generic non-linear optimization routine FMINCON solver has been used as a classical optimization which implements the SQP (Sequential Quadratic Programming) and the interior-point method for optimization of LFC problem.

\subsection{Sensitivity analyses}

In order to analyze the effects of synchronous power control gains, $\left(\omega_{n 1}, \zeta_{1}, \omega_{n 2}\right.$, and $\left.\zeta_{2}\right)$, on the system behavior, numerical simulation is performed with different values of control gains in VSP controllers. These analyses are performed for two-area system, considering the data of Tables $1-3$, under a large load step-change

Table 1

GENCOs parameters.

\begin{tabular}{llllll}
\hline \multirow{2}{*}{ Parameters } & Area1 & & & Area2 & \\
\cline { 2 - 3 } \cline { 5 - 6 } & GENCO1 & GENCO2 & & GENCO3 & GENCO4 \\
\hline$T_{t}(\mathrm{~s})$ & 0.32 & 0.30 & & 0.30 & 0.32 \\
$T_{g}(\mathrm{~s})$ & 0.06 & 0.08 & & 0.06 & 0.07 \\
$R($ Hz/p.u. $)$ & 2.4 & 2.5 & & 2.5 & 2.7 \\
\hline
\end{tabular}

Table 2

Control area parameters.

\begin{tabular}{lll}
\hline Parameters & Area1 & Area2 \\
\hline$K_{P}($ Hz/p.u.) & 102 & 102 \\
$T_{P}($ s $)$ & 20 & 25 \\
$\beta$ (p.u./Hz) & 0.425 & 0.396 \\
$T_{12}$ (p.u./rad) & 0.245 & \\
\hline
\end{tabular}

Table 3

Control parameters of the studied AC/DC model with VSP.

\begin{tabular}{lrc}
\hline \multicolumn{2}{l}{ Parameters } & Value \\
\hline \multicolumn{2}{l}{$K_{f 1, \text { vSP }}$ (p.u. MW/rad) } & 3.5 \\
$K_{f 2, \text { vSP }}$ (p.u. MW/rad) & -1.61 \\
$K_{\text {AC,VSP }} \quad$ (p.u. MW) & 3.1 \\
$\omega_{n 1}$ & (rad/s) & 6.9 \\
$\omega_{n 2}$ & $(\mathrm{rad} / \mathrm{s})$ & 0.022 \\
$\zeta_{1}$ & (p.u.) & 1.30 \\
$\zeta_{2}$ & (p.u.) & 1.01 \\
\hline
\end{tabular}

around 0.03 p.u at $3.5 \mathrm{~s}$ in the Area1. The sensitivity analysis is explained and presented as a classical method for analyzing the effect of each VSP over the system behavior. Therefore, the proper ranges for each parameters are obtained and discussed in this section.

\subsubsection{Effects of variations in VSP1 parameters}

As explained before, the VSP1 has two important parameters, $\zeta_{1}$ and $\omega_{n 1}$. The effect of $\omega_{n 1}$ from VSP1 on the system behavior is presented in Figs. 7 and 8 while the parameters of the VSP2 are the same as the ones presented in Table 3. The value of $\omega_{n 1}$ is increasing from 0.3 to 7 and the system responses are analyzed. The frequency deviations and output power generations are presented in Figs. 7 and 8, respectively. It is clear that the dynamic performance can be improved by increasing the $\omega_{n 1}$ factor of VSP1. These results are more clear comparing the results presented in Figs. 9 and 10, when after increasing the $\omega_{n 1}$ the peak value is decreasing and at the same time, lower settling time is achievable.

The effects of damping parameter $\left(\zeta_{1}\right)$ in VSP1 is presented in Figs. 11 and 12 . The damping factor has changed from 0.08 to 2.6 and the system response for each situation is presented and compared. As shown in Fig. 11, lower values of damping will result in various oscillations in the frequency of Area1, while very high values make the system response more slow (higher settling time).

Therefore, for having a suitable dynamic response, a proper damping is necessary. But comparing the results in Fig. 12, shows that the best value for damping is around 1.3 which will bring the best dynamic performance with less oscillation and minimum settling time is achieved.

The dynamic response of emulated power by VSP1 $\left(\Delta x_{1, \mathrm{VSP} 1}\right)$ is also presented in Fig. 13. It is obvious that lower damping will bring lots of oscillation which is totally unacceptable. But by increasing the damping of VSP1 better dynamic response could be achieved by VSP1.

\subsubsection{Effects of variations in VSP2 parameters}

The effect of second synchronous power controller, which is located in the second area is presented. These parameters are inertia gain $\left(\omega_{n 2}\right)$ and damping $\left(\zeta_{2}\right)$. The parameters of the VSP1 are the same as the ones presented in Table 3 and the effects of variation in VSP2 is analyzed here. The frequency deviations for different values of $\omega_{n 2}$ are presented in Figs. 14 and 15.

Increasing the $\omega_{n 2}$ value will lead to more participation from VSP2 compared to VSP1, but since in the studied system the load contingency is in area one, this increase will not have a positive effect on the overall system behavior. Therefore, the lower value of $\omega_{n 2}$ in VSP2 is expectable. As shown in the figures, the value of $\omega_{n 2}$ is increasing from 0.02 to 8 . It is observed that the lower values for $\omega_{n 2}$ is preferable. The higher values of $\omega_{n 2}$ is leading to high overshoot. 

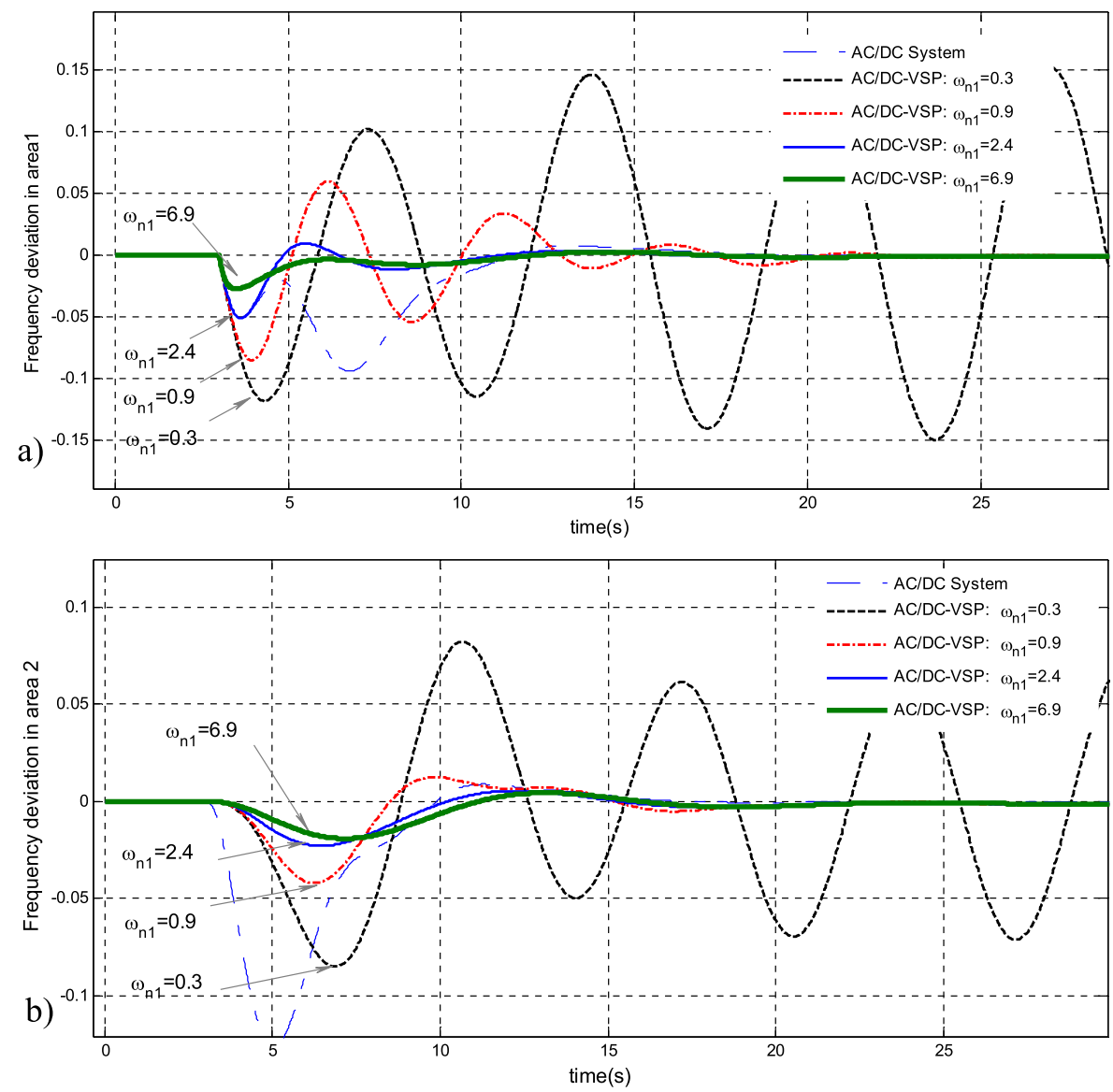

Fig. 7. Frequency deviations in Area 1 and 2 for difference control gain values.
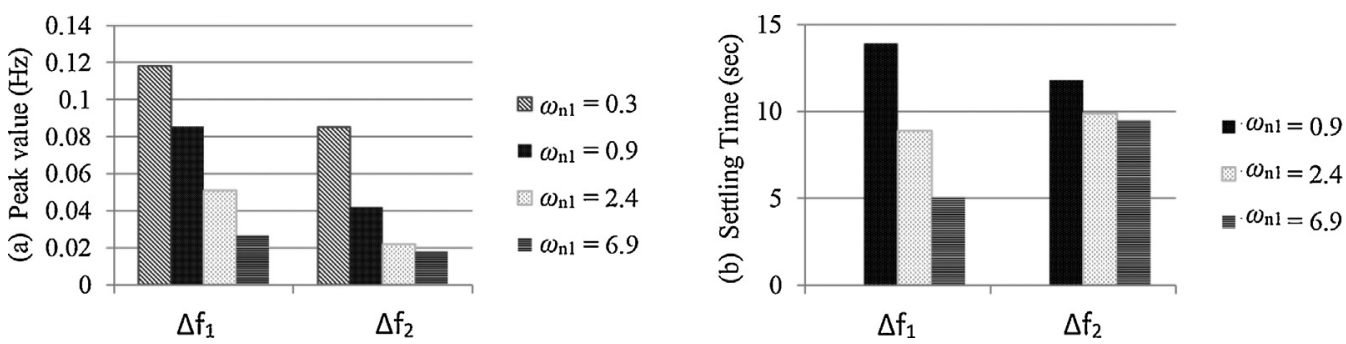

Fig. 8. Frequency characteristics: (a) peak overshoot, and (b) settling time

The effects of damping parameter $\left(\zeta_{2}\right)$ in VSP2 is also presented in Figs. 16 and 17. The damping factor has changed from 0.3 to 2 and the system response for each situation is presented and compared.

As it was expected, damping value is necessary to reach better dynamic performance. As shown in Fig. 16, lower value of damping in VSP2 is leading to steady state error with unacceptable performance.

Fig. 17, is also presenting the response of the power state emulated by VSP2 ( $\Delta x_{1}$,VSP2 $)$ for different values of damping. As shown in Fig. 17, higher values for damping in VSP2 will bring a better response with less time response.

\subsection{Eigenvalue sensitivities}

In order to perform eigen sensitivity analyses, the matrix of participation factor should be calculated. The participation matrix for two-area studied power system is calculated in this section. Based on the obtained result, it can be observed that exactly which states have more participation in sensitive or critical modes. Here all the information about participation matrix is presented. The same as before, this information will be useful to identify the participant state for each sensitive mode.

Participation factor matrix:

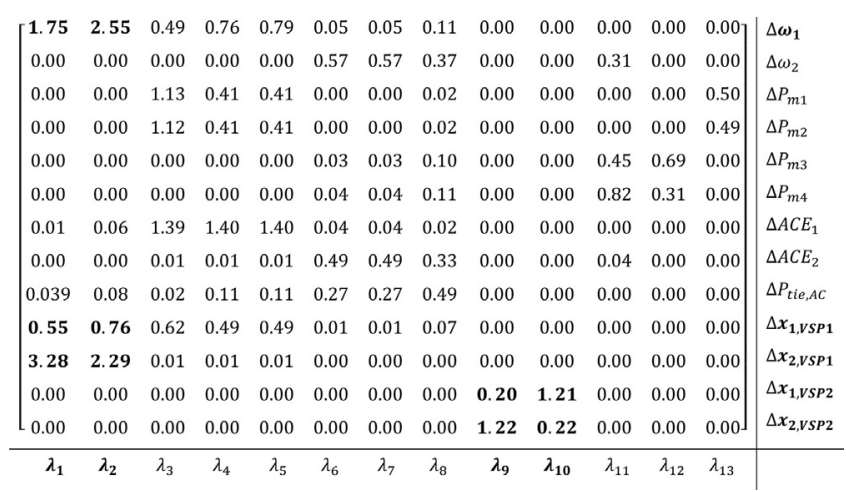



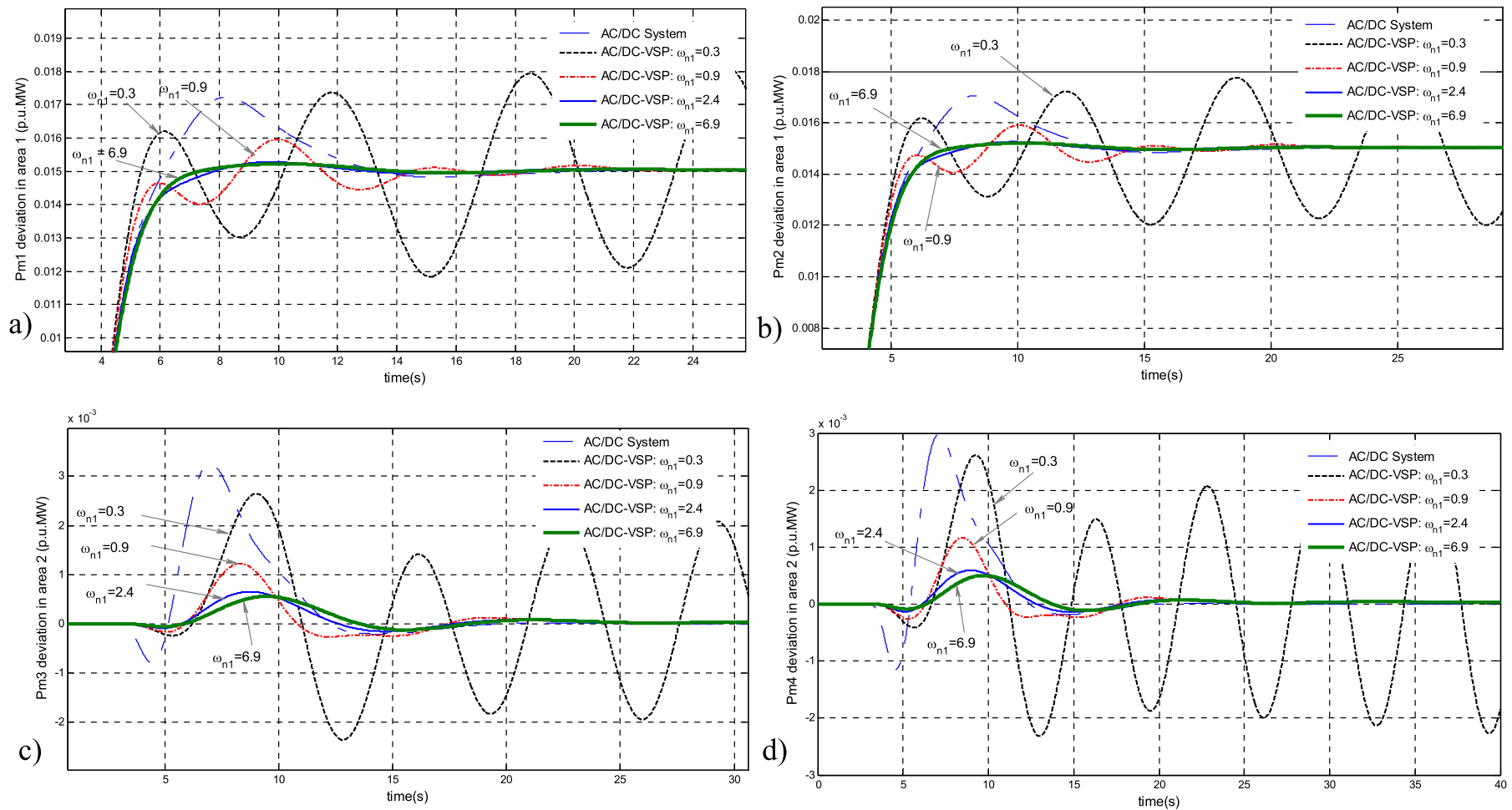

Fig. 9. Active power generations. (a) GENCO1, (b) GENCO2, (c) GENCO3, (d) GENCO4.

Another objective in eigenvalue sensitivity analysis is to identify the sensitivity of eigenvalues to each element of state matrix $A$ in the system. As it was explained before, the sensitivity matrix of the elements in matrix $A$ could be as follows:

$P_{\text {sens }}=\Psi_{i k} \Phi_{j i}=\frac{\partial \lambda_{i}}{\partial a_{k j}}$

where the sensitivity of the eigenvalue $\lambda_{i}$ to the element $a_{k j}$ of the state matrix $A$ will be equal to the product of the left eigenvector element $\Psi_{i k}$ and the right eigenvector element $\Phi_{j i}$. This tells us that the best way to change the ith mode is to apply a control to the state variable such that the above sensitivity has the largest participating factor.

In order to apply such analysis for the studied two-area power system, the state matrix $A$ of studied two-area system with VSP is partitioned as presented by Eqs. (32) and (33).

As identified in state space presentation of global system in Eq. (32), the parameters of synchronous power control are appeared in sub-matrices $A_{21}$ which are related to VSP control state variables. In fact, these parameters are presented in 11th and 13th rows of the global system matrix $A$ and could be used in analyzing the system performance. Therefore, the elements of interest in matrix
Table 4

Normalized sensitivity of each mode for important elements of $A$ matrix.

\begin{tabular}{llllll}
\hline Sensitivity of $\lambda_{i}$ & $a_{11,1}$ & $a_{11,2}$ & $a_{11,9}$ & $a_{11,10}$ & $a_{11,11}$ \\
\hline$\lambda_{1}$ & 0.3120 & 0.0003 & 0.0003 & 0.065 & 1.001 \\
$\lambda_{2}$ & 0.3138 & 0.0007 & 0.0005 & 0.0679 & 0.697 \\
$\lambda_{3}$ & 0.0020 & 0.000 & 0.000 & 0.0036 & 0.0036 \\
$\lambda_{4}$ & 0.0044 & 0.0003 & 0.000 & 0.0035 & 0.0036 \\
$\lambda_{5}$ & 0.0044 & 0.0003 & 0.000 & 0.0035 & 0.0036 \\
$\lambda_{6}$ & 0.0020 & 0.0029 & 0.000 & 0.000 & 0.000 \\
$\lambda_{7}$ & 0.0020 & 0.0029 & 0.000 & 0.000 & 0.000 \\
$\lambda_{8}$ & 0.0011 & 0.0016 & 0.000 & 0.0004 & 0.000 \\
$\lambda_{9}$ & 0.0004 & 0.0004 & 0.000 & 0.000 & 0.000 \\
$\lambda_{10}$ & 0.0004 & 0.0004 & 0.000 & 0.000 & 0.000 \\
$\lambda_{11}$ & 0.000 & 0.000 & 0.000 & 0.000 & 0.000 \\
$\lambda_{12}$ & 0.000 & 0.000 & 0.000 & 0.000 & 0.000 \\
$\lambda_{13}$ & 0.000 & 0.000 & 0.000 & 0.000 & 0.000 \\
\hline
\end{tabular}

$A$ are the elements which contain the control gains of VSP control components $\left(\omega_{n 1}, \omega_{n 2}, \zeta_{1}\right.$ and $\left.\zeta_{2}\right)$ in sub-matrix $A_{21}$.

The results of sensitivities to the state matrix $A_{(12 \times 12)}$ parameters are presented by Tables 4 and 5 . In these tables the absolute value for sensitivity of each mode to the elements of sub-matrix $A_{21}$, which are of our interest, is presented.
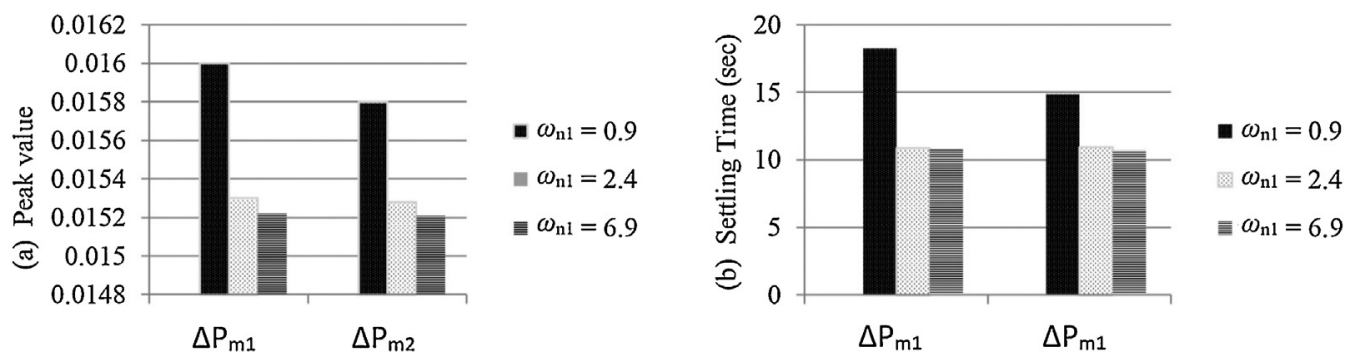

Fig. 10. Settling times of active power response for GENCO1 and GENCO2. 

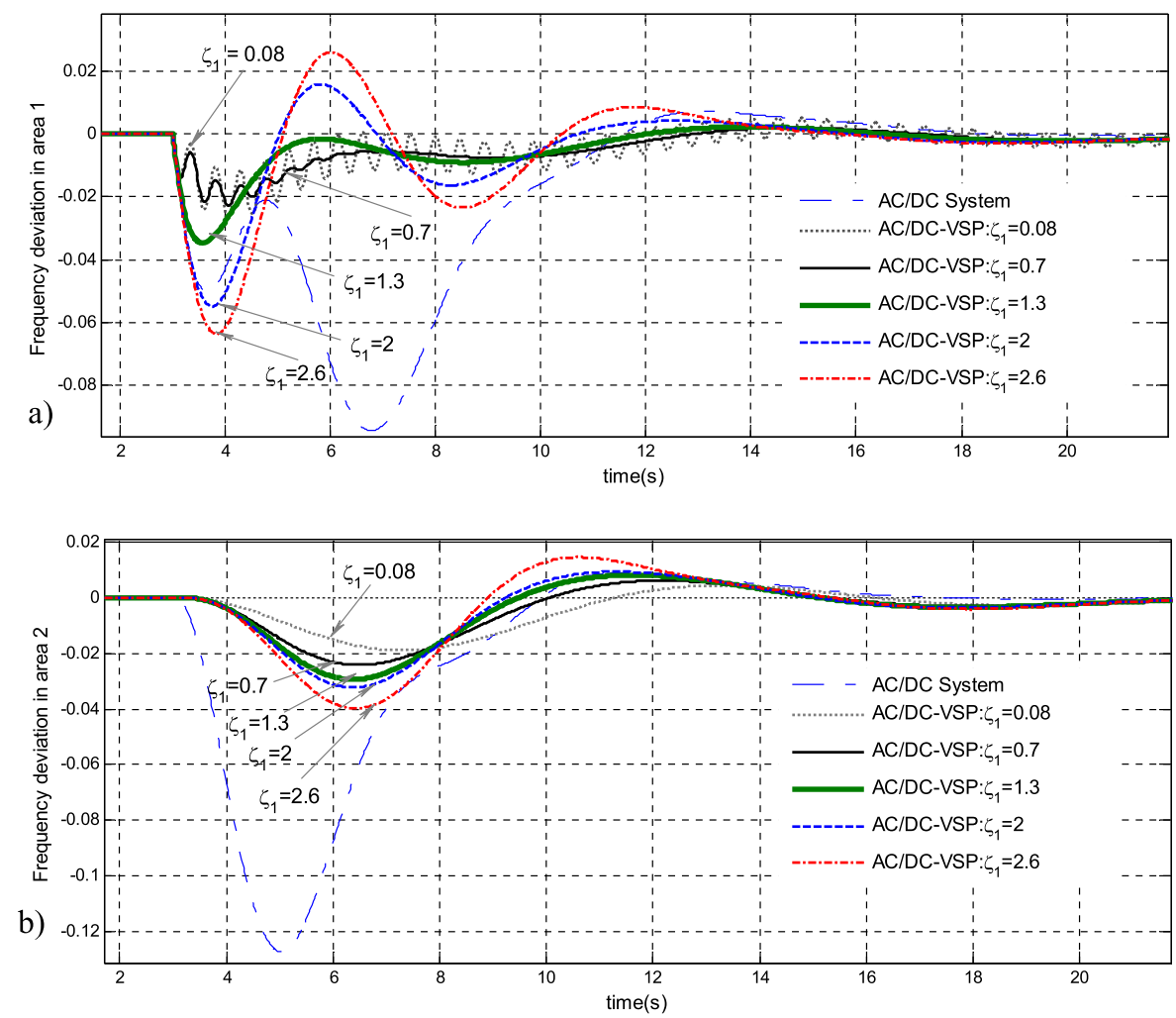

Fig. 11. Frequency deviations for different damping in VSP1, (a) Area1, (b) Area2.
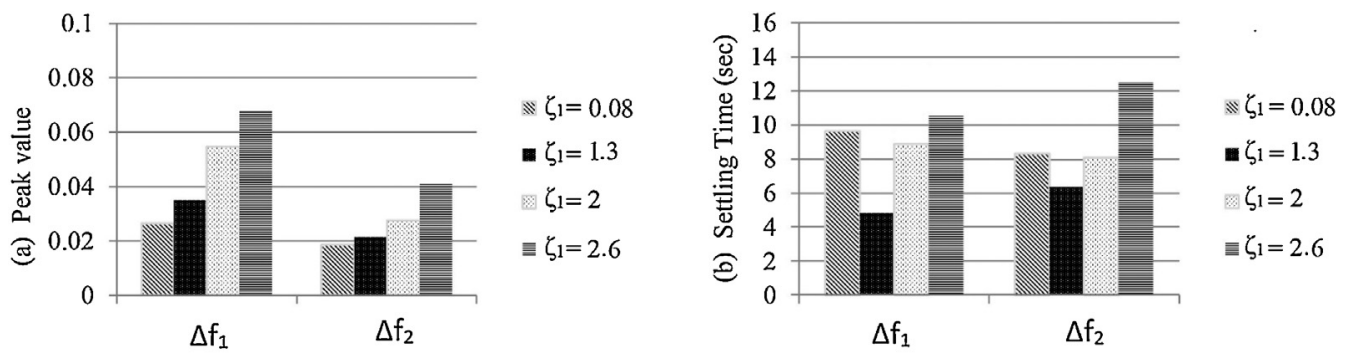

Fig. 12. Frequency characteristics: (a) peak overshoot, and (b) settling time for $1 \%$ of the final value.

From the presented information in Table 4 , it is obvious that the sensitivities for $a_{11,1}$ and $a_{11,11}$ are higher than other elements. For these elements of matrix $A$, the first and second modes $\left(\lambda_{1}\right.$ and $\lambda_{2}$ ) have the most sensitivities. From participation factor matrix it could be observed that these modes are related to the first, 10th and 11 th states of the system $\left(\Delta \omega_{1}, \Delta x_{1, \mathrm{VSP} 1}\right.$ and $\left.\Delta x_{2, \mathrm{VSP} 1}\right)$.

The same as before, the sensitivities to the rest of elements are presented in Table 5. These parts of elements are the ones from matrix $A_{21}$ which contain VSP2 parameters presented by Eq. (33):

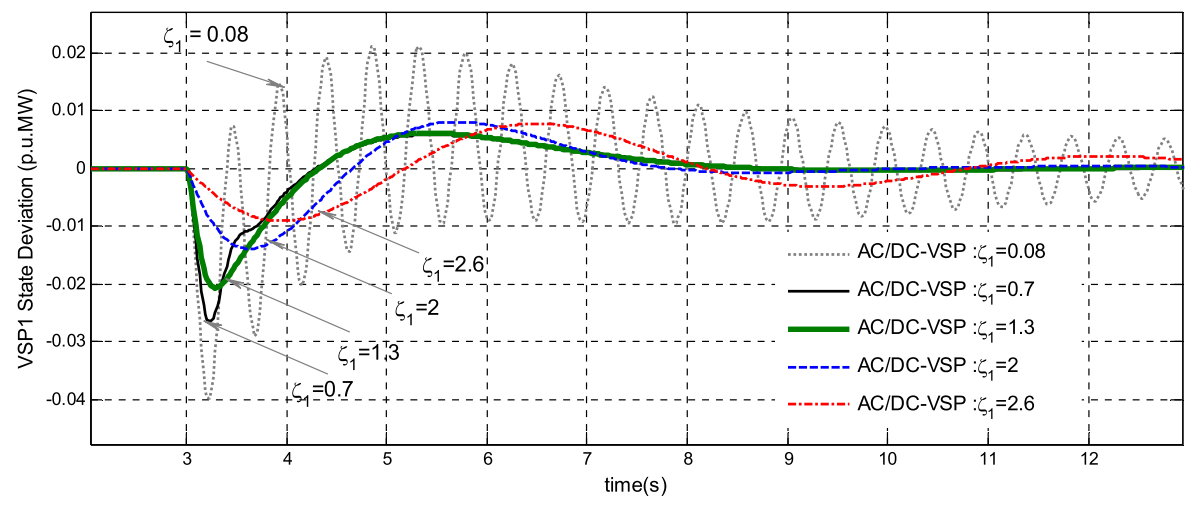

Fig. 13. Emulated power by VSP1. 

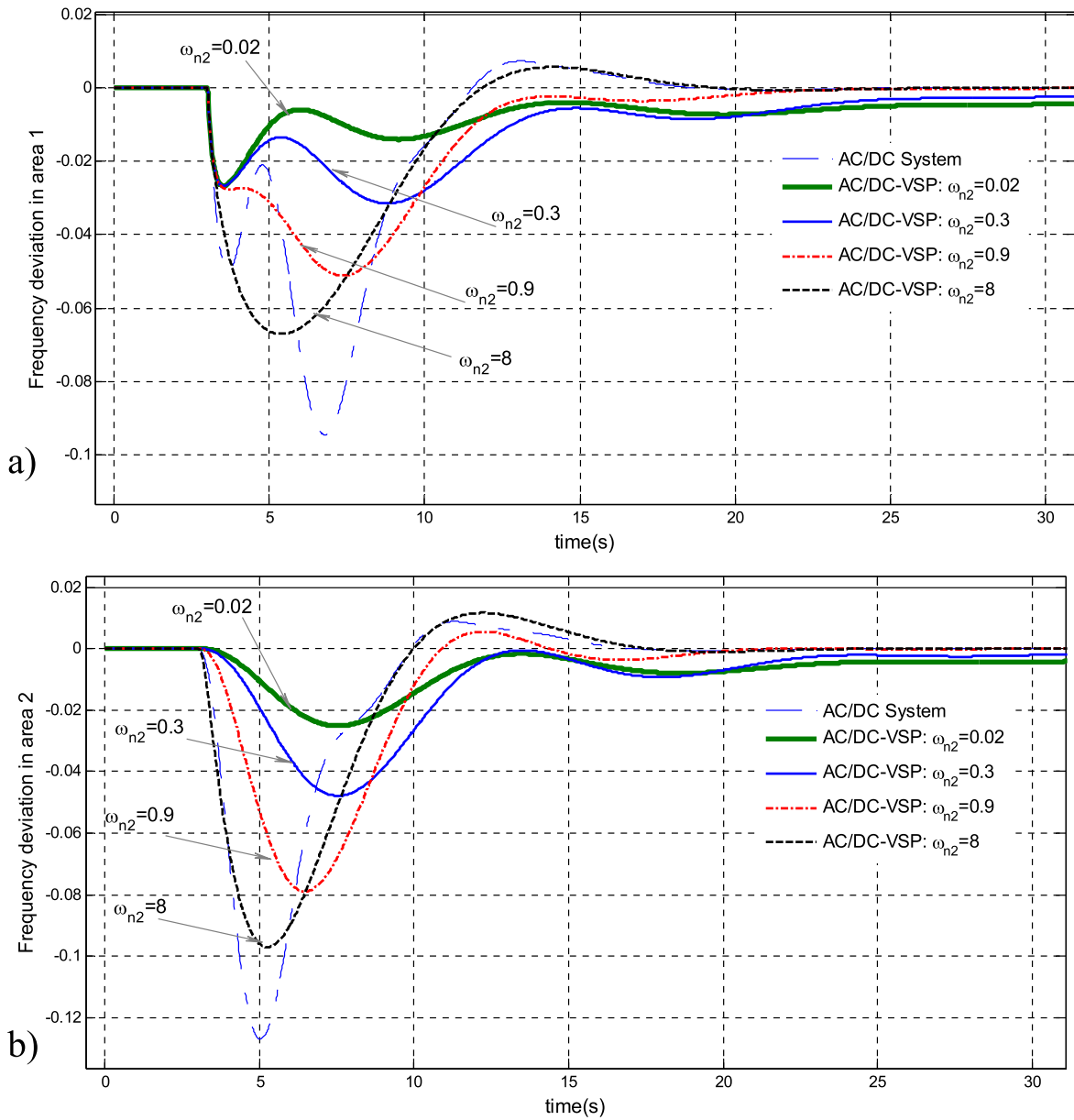

Fig. 14. Frequency deviations in Area 1 and 2 for difference control gain values.
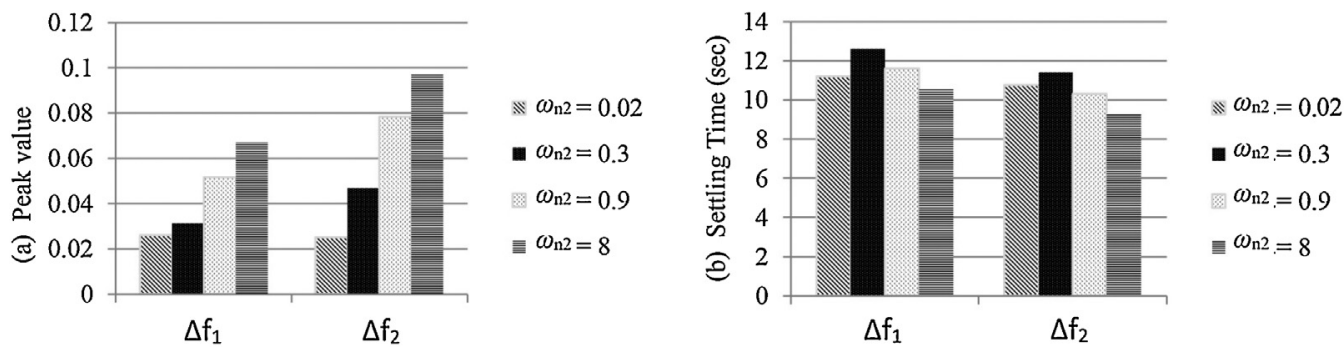

Fig. 15. Frequency characteristics: (a) peak overshoot, and (b) settling time for $1 \%$ of the final value.

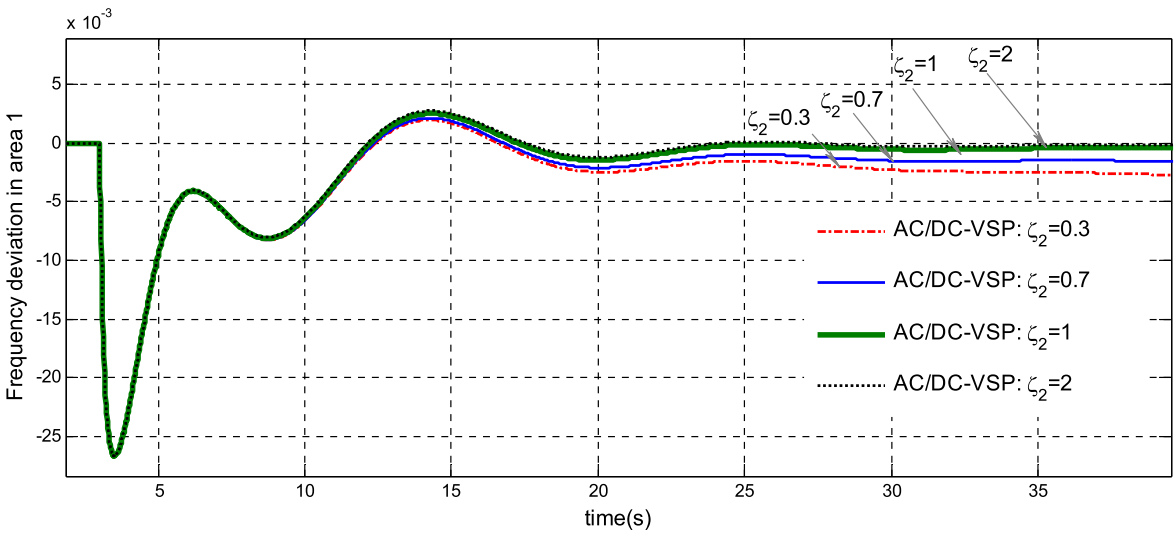

Fig. 16. Frequency deviations in Area 1 for different damping in VSP2. 


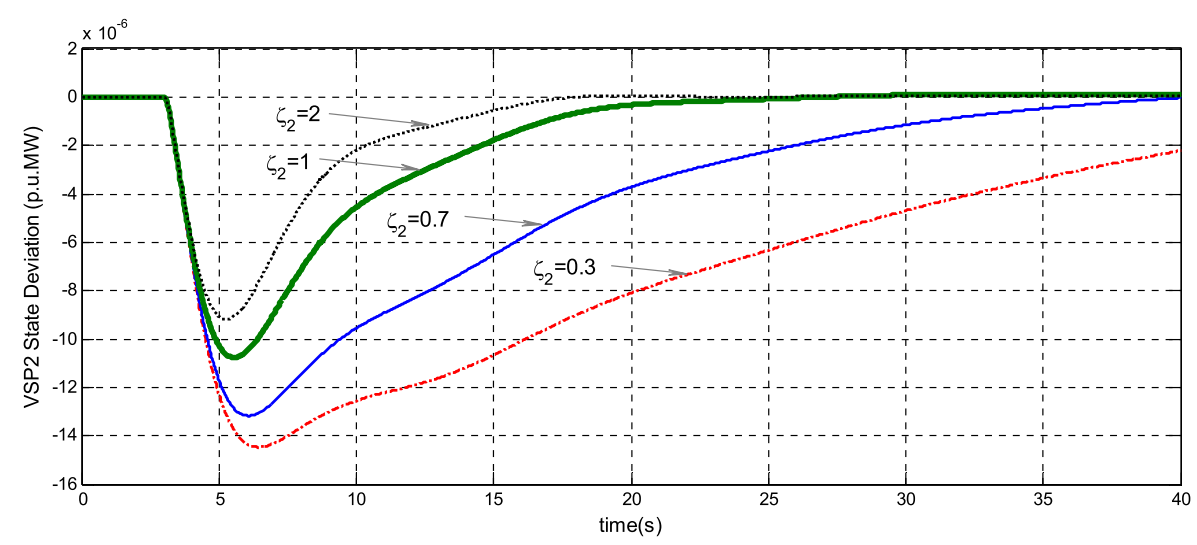

Fig. 17. Emulated power by VSP2 for different damping.

From the presented information in Table 5, it can be observed that most of the elements have a considerable sensitivities to 9th and 10th modes $\left(\lambda_{9}\right.$ and $\left.\lambda_{10}\right)$. From the participation factor matrix it can be indicated that the 12th and 13th states ( $\Delta x_{1, \text { VSP2 }}$ and $\left.\Delta x_{2, \mathrm{VSP} 2}\right)$ have the main participation for these two modes.

The summary of the eigenvalue sensitivity analysis is presented in Table 6 . As shown by sensitivity matrices in Tables 4 and $5, \lambda_{1}$, $\lambda_{2}, \lambda_{9}$ and $\lambda_{10}$ have the main sensitivity. Considering the results of participation matrix, it is indicated that controlling the 10th, 11th, 12th, and 13th states (the states of both VSP controllers) are the most important ones for control design.

As explained before, the VSP states ( $\Delta x_{1, \mathrm{VSP} 1}, \Delta x_{2, \mathrm{VSP} 1}, \Delta x_{1, \mathrm{VSP} 2}$ and $\left.\Delta x_{2, \mathrm{VSP} 2}\right)$ are affected by their control gains. So with proper selection of control gains, the desired dynamic response could be obtained.

In the last part of the analyses, for investigating the effects of VSP based HVDC parameters on the system performance and to identify their proper ranges, another analysis for modal characteristics (like damping and frequency of oscillatory modes) of the studied system regarding parameter variation of VSP stations are performed as below. The relationship between the $\omega_{n}$ gains $\left(\omega_{n 1}\right.$ and $\left.\omega_{n 2}\right)$ of synchronous controller and the damping of critical modes is presented in Fig. 18.

Table 5

Normalized sensitivity of each mode for important elements of $A$ matrix.

\begin{tabular}{llllll}
\hline Sensitivity of $\lambda_{i}$ & $a_{13,1}$ & $a_{13,2}$ & $a_{13,9}$ & $a_{13,12}$ & $a_{13,13}$ \\
\hline$\lambda_{1}$ & 0.0012 & 0.000 & 0.000 & 0.000 & 0.000 \\
$\lambda_{2}$ & 0.0025 & 0.000 & 0.000 & 0.000 & 0.000 \\
$\lambda_{3}$ & 0.000 & 0.000 & 0.000 & 0.000 & 0.000 \\
$\lambda_{4}$ & 0.0002 & 0.000 & 0.000 & 0.000 & 0.000 \\
$\lambda_{5}$ & 0.0002 & 0.0026 & 0.000 & 0.000 & 0.000 \\
$\lambda_{6}$ & 0.0301 & 0.0435 & 0.0025 & 0.000 & 0.000 \\
$\lambda_{7}$ & 0.0301 & 0.0435 & 0.0025 & 0.000 & 0.000 \\
$\lambda_{8}$ & 0.0035 & 0.0053 & 0.0007 & 0.000 & 0.000 \\
$\lambda_{9}$ & 0.9920 & 0.9001 & 0.0941 & 0.1529 & 0.0076 \\
$\lambda_{10}$ & 1.001 & 0.9810 & 0.0941 & 0.1529 & 0.00135 \\
$\lambda_{11}$ & 0.0004 & 0.00122 & 0.000 & 0.000 & 0.000 \\
$\lambda_{12}$ & 0.000 & 0.000 & 0.000 & 0.000 & 0.000 \\
$\lambda_{13}$ & 0.000 & 0.000 & 0.000 & 0.000 & 0.000 \\
\hline
\end{tabular}

Table 6

Summary of eigen sensitivity analysis.

\begin{tabular}{ll}
\hline Sensitive modes to $A$ matrix elements & Participant states \\
\hline$\lambda_{1}$ & $\Delta x_{1, \mathrm{VSP} 1}, \Delta x_{2, \mathrm{VSP} 1}, \Delta \omega_{1}$ \\
$\lambda_{2}$ & $\Delta x_{1, \mathrm{VSP} 1}, \Delta x_{2, \mathrm{VSP} 1}, \Delta \omega_{1}$ \\
$\lambda_{9}$ & $\Delta x_{1, \mathrm{VSP} 2}, \Delta x_{2, \mathrm{VSP} 2}$ \\
$\lambda_{10}$ & $\Delta x_{1, \mathrm{VSP} 2}, \Delta x_{2, \mathrm{VSP} 2}$ \\
\hline
\end{tabular}

The 3-D presentation in Fig. 18, will indicates the area with highest damping. As shown in this figure, the highest damping will achieve for high values of $\omega_{n 1}$ and low values for $\omega_{n 2}$. It means, for the studied two-area case, the values for $\omega_{n 1}$ should be higher than 5 and the values for $\omega_{n 2}$ should be lower than 1 . These results are exactly the same as the results which were obtained from the eigenvalue analyses presented before.

For investigating the proper range of damping in VSP1, another plot is presented in Fig. 19. In this figure, the relationships between parameters of VSP1 $\left(\omega_{n 1}\right.$ and $\left.\zeta_{1}\right)$ and system damping is also presented. As shown in this figure, the highest damping is achieved for the space with damping value higher than 1 and lower than 3 . For the other parameter of VSP1, $\omega_{n 1}$, the range is the same as the one presented in Fig. 18.

For investigating the proper range of damping in the second VSP controller, another plot is presented in Fig. 20. In this figure, the relationships between damping ratio parameters of both VSP controllers $\left(\zeta_{1}\right.$ and $\left.\zeta_{2}\right)$ and system damping is presented.

As shown in Fig. 20, the highest damping in the system modes is achieved for the area with the values higher than 0.5 for $\zeta_{2}$. The proper range for the other damping ratio, $\zeta_{1}$, is the same as the one from Fig. 19.

Therefore, based on the presented analysis, the acceptable range of VSP parameter variations which can guaranty a stable and

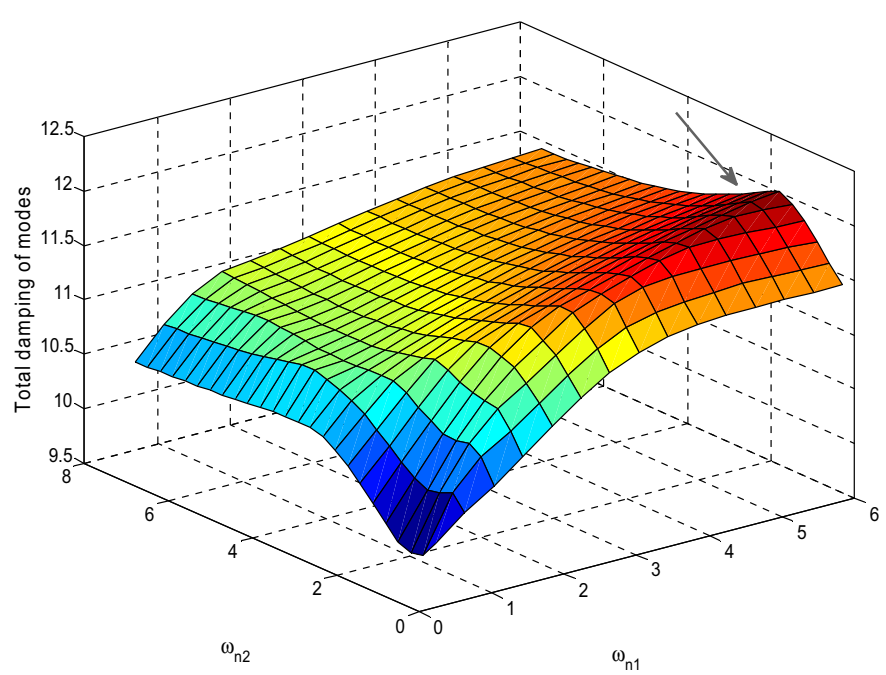

Fig. 18. 3-D presentation for damping of critical modes for different values of $\omega_{n 1}$ and $\omega_{n 2}$. 


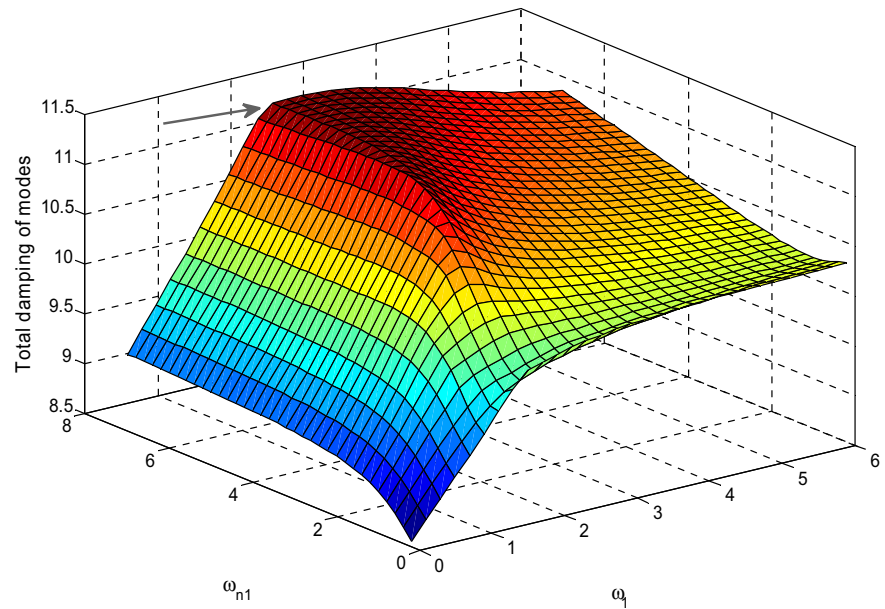

Fig. 19. 3-D presentation for damping of critical modes for different values of $\omega_{n 1}$ and $\zeta_{1}$.

acceptable dynamic response in the studied case could be as follows:

$\omega_{n 1}>5$

$\omega_{n 2}<1$

$1<\zeta_{1}<3$

$\zeta_{2}>0.5$

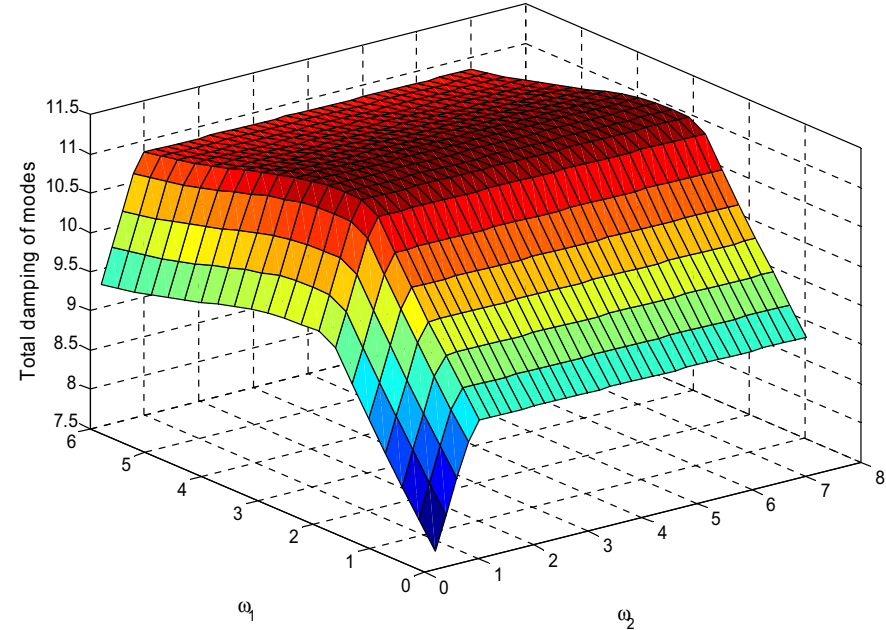

Fig. 20. 3-D presentation for damping of critical modes for different values of $\zeta_{1}$ and $\zeta_{2}$.

\subsection{Comparison and simulation results}

In order to validate and evaluate the positive effects of proposed VSP-based AC/DC system on AGC dynamics and to show how the proposed approach can help the system dynamic during contingencies, a common scenario for two-area test system which was explained in the previous sections is considered. It is assumed the contingency is a load variation in Area 1 around 0.03 p.u. at 3 s. All the analysis and simulations are performed in Matlab platform.

Several comparisons are performed during this simulation. Comparisons are related to the normal AC system, the typical $\mathrm{AC} / \mathrm{DC}$ system and the proposed model for AC/DC system with VSP based inertia emulation. Considering these simulations, the
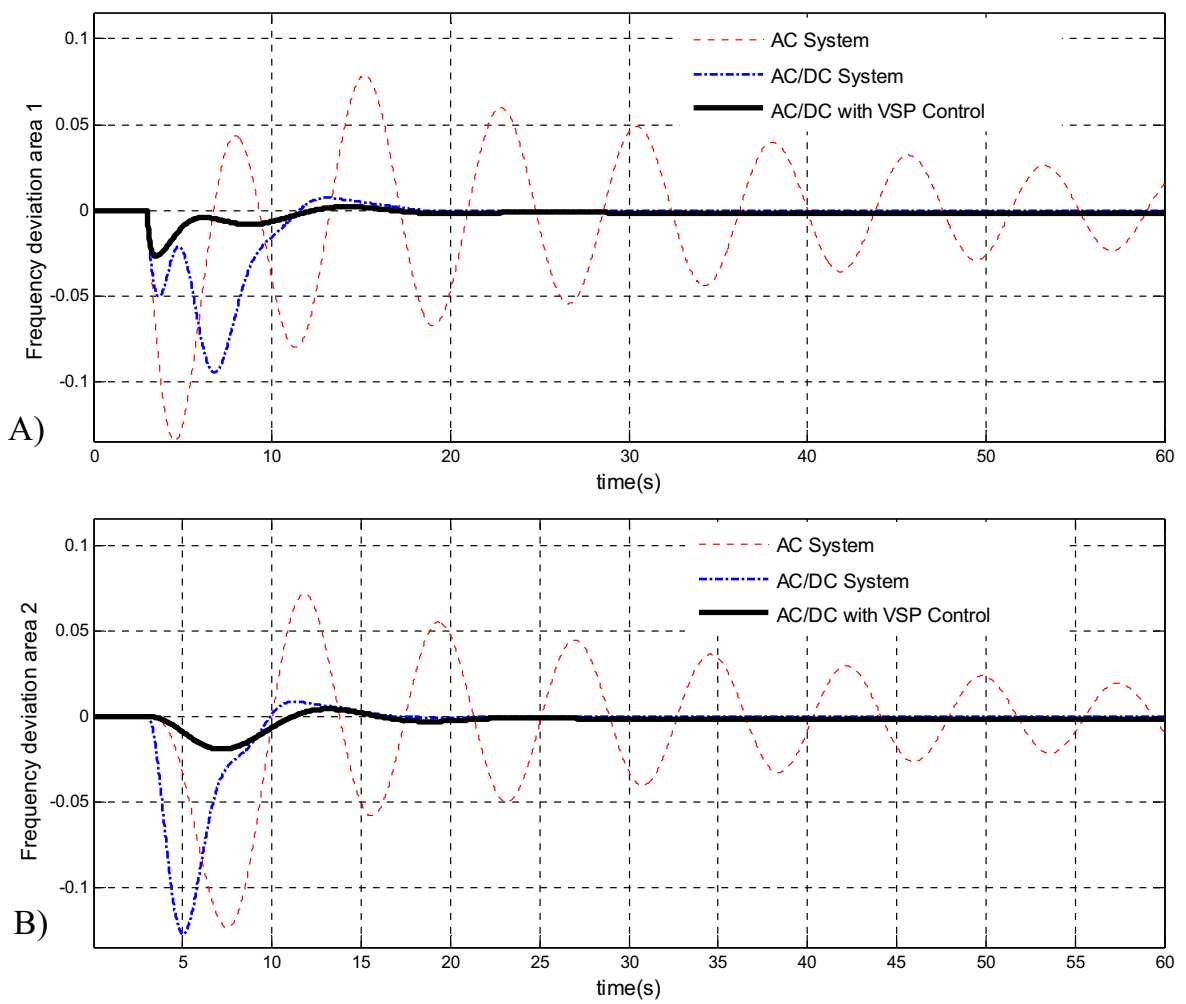

Fig. 21. Dynamic response of frequency deviations; (A) Area1, (B) Area2. 

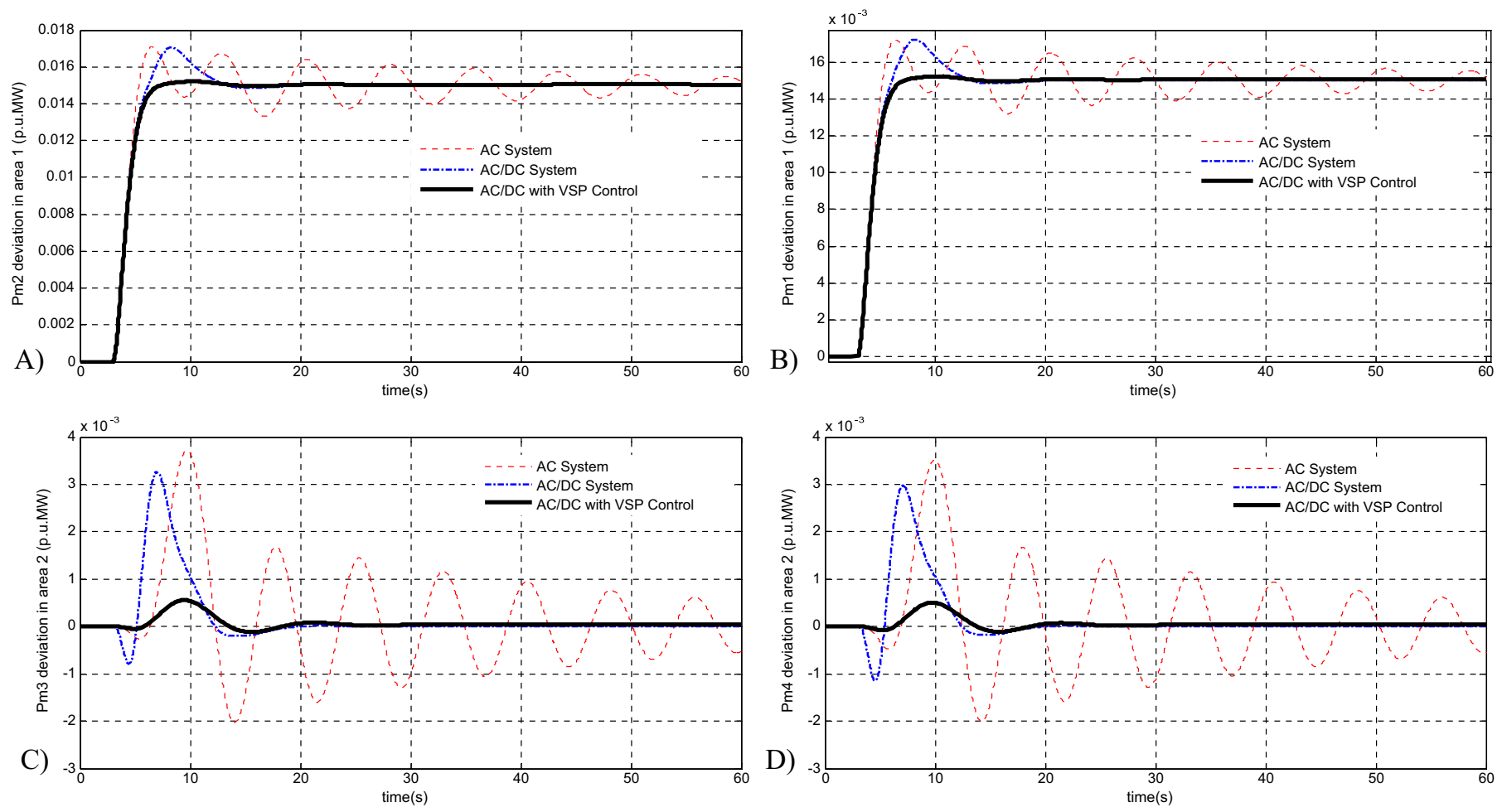

Fig. 22. Output power generation; (A) GENCO1, (B) GENCO2, (C) GENCO3, and (D) GENCO4.

positive effects of VSP method will be identified. The Frequency deviations in both areas are presented in Fig. 21.

The output power generated by each unit is also presented in Fig. 22. It is clear that by means of inertia emulation it would be possible to change the dynamic response of system and the final response will be smoother than the normal system without virtual inertia capabilities. It is clear that the synchronous power control technique has a better performance with several advantages for emulating the inertia.

As it was discussed before, in the VSP technique there is no need to PLL and frequency estimation and considering the fact that simultaneous damping and inertia could be emulated, a powerful method for improving the system dynamics during the contingencies is proposed.

The dynamic behaviours of emulated power for VSP inertia emulation are also presented in Fig. 23. The comparisons are made between two controllers in different area. The main contribution is coming from the one in Area1 (area with contingency). Since the

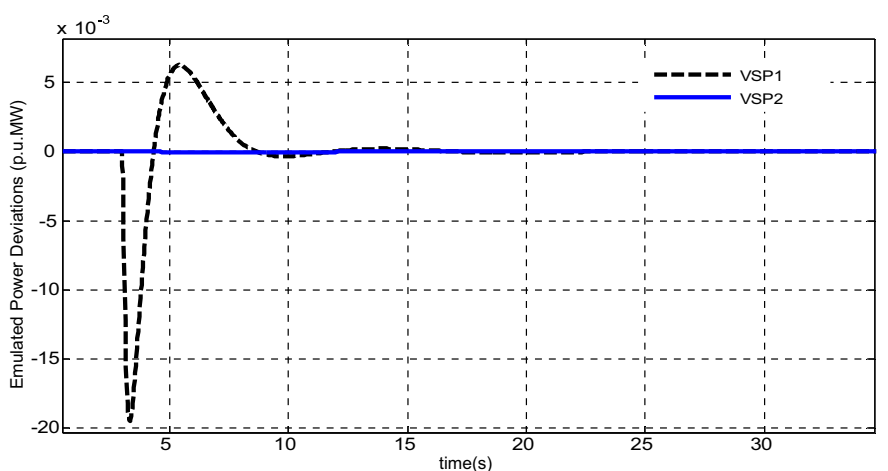

Fig. 23. Comparisons of emulated power deviations for VSP1 in Area 1 and VSP2 in Area2. load change is happening in Area1, it is predictable that the controller in Area1 will have more contribution compared to the second controller in Area2. As shown in Fig. 23, the maximum emulated power is less than $2 \%$ of the rated power.

Another comparison regarding eigenvalue of the system considering different systems is presented in Table 7. It is clear that the method based on synchronous power strategy has better performance, less oscillatory modes with higher damping is achievable.

\subsection{Case study with 39-bus test system}

To investigate the performance of the proposed control strategy with a more complex system, a network with more elements as the well-known IEEE 10 generators 39-bus system is considered as the test system. This system is widely used as a standard system for testing of new power system analysis and control synthesis methodologies. Fig. 24 shows a single-line diagram of the test system.

Table 7

Eigenvalue comparisons for different two-area interconnected systems.

\begin{tabular}{llll}
\hline Modes & AC system & AC/DC system & AC/DC with VSP method \\
\hline$\lambda_{1}$ & $-0.027+0.825 i$ & -23.9299 & -15.1962 \\
$\lambda_{2}$ & $-0.027-0.825 i$ & $-0.4945+1.6611 i$ & -10.2239 \\
$\lambda_{3}$ & $-0.342+0.399 i$ & $-0.4945-1.6611 i$ & -1.4311 \\
$\lambda_{4}$ & $-0.342-0.399 i$ & $-0.3437+0.4245 i$ & $-0.9586+0.4913 i$ \\
$\lambda_{5}$ & -0.7023 & $-0.3437-0.4245 i$ & $-0.9586-0.4913 i$ \\
$\lambda_{6}$ & -1.8271 & -0.8974 & $-0.1916+0.5618 i$ \\
$\lambda_{7}$ & -2.1073 & $-1.9374+0.1752 i$ & $-0.1916-0.5618 i$ \\
$\lambda_{8}$ & -2.6872 & $-1.9374-0.1752 i$ & -0.6072 \\
$\lambda_{9}$ & -2.6316 & -2.6850 & -0.473 \\
$\lambda_{10}$ & - & -2.6316 & -0.088 \\
$\lambda_{11}$ & - & - & -2.1129 \\
$\lambda_{12}$ & - & - & -2.6873 \\
$\lambda_{13}$ & - & - & -2.6316 \\
\hline
\end{tabular}




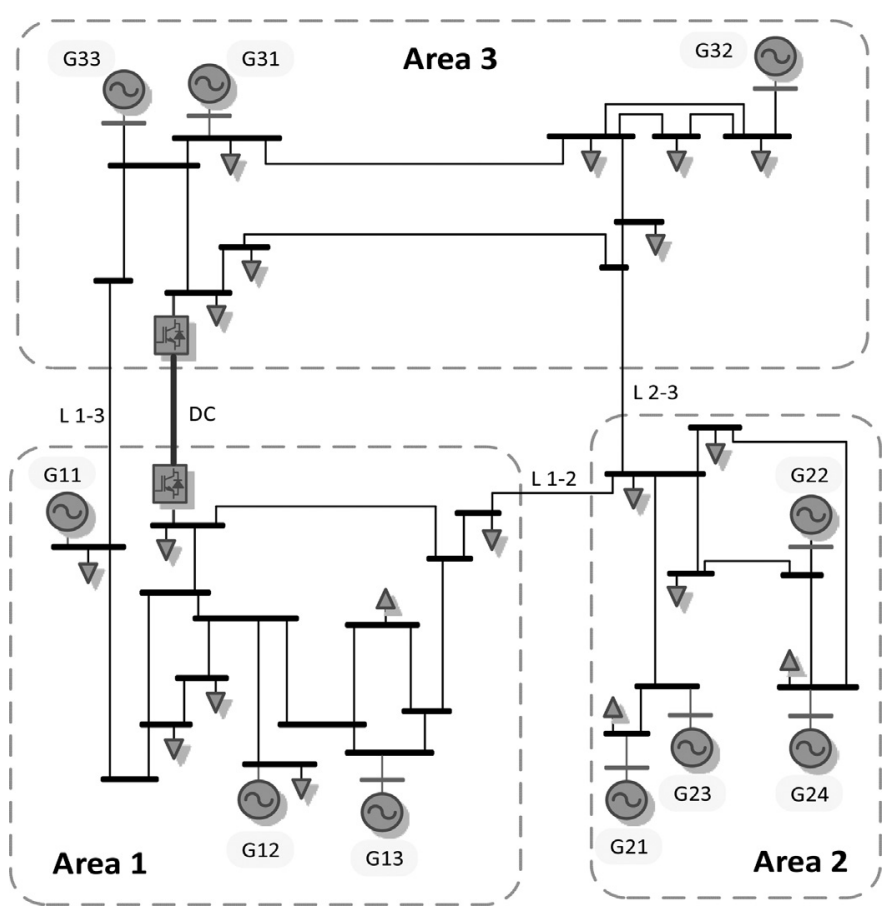

Fig. 24. The configuration of the three-area power system with HVDC link.

This system consists of 3 areas, 10 generators and the system parameters are shown in Table 8 [21]. The total load in this system is assumed to be $5.483 \mathrm{GW}$ for the base system of $100 \mathrm{MVA}$ and $60 \mathrm{~Hz}$. More details about this system data can be found in Refs. [21-23]. As mentioned, the main objective of this paper is to propose an effective LFC scheme with a desirable performance in the presence of the VSP based HVDC system. Therefore, the case study is updated by adding a VSP based HVDC line between Area1 and 3. As shown in Fig. 24, the tie-line flows are used for control studies. In this simulation, the important inherent requirement such as governor dead band and generation rate constraint imposed by physical system dynamics are considered.

In this scenario, it is assumed that the main contingency happened as a load change in Area3 by increasing to 0.1 p.u. at $t=3 \mathrm{~s}$. All generators participate in LFC with equal participation factors. In the simulation, the parameters of the VSP based HVDC system are obtained using optimization theory as explained in previous sections. These values are presented in Table 9.

It is worth to mention that, it is not necessary to equip all the converters with the VSP control during AGC task. In fact, the number of converters which are facilitated by VSP control will be depended on grid topology and specific conditions and requirements of each area in terms of ancillary services. In our work, we assumed that

Table 8

Parameters of test system. (Base power of $100 \mathrm{MVA}$ ).

\begin{tabular}{lllllll}
\hline Area & GENCO & $H$ & $X_{d}$ & $X_{q}$ & $T_{\text {do }}$ & $X_{l}$ \\
\hline 1 & G11 & 70.0 & 0.02 & 0.019 & 7.0 & 0.003 \\
& G12 & 30.3 & 0.295 & 0.282 & 1.5 & 0.035 \\
& G13 & 35.8 & 0.249 & 0.237 & 1.5 & 0.030 \\
2 & G21 & 28.6 & 0.262 & 0.258 & 1.5 & 0.029 \\
& G22 & 26.0 & 0.67 & 0.620 & 0.44 & 0.054 \\
& G23 & 34.8 & 0.254 & 0.241 & 0.4 & 0.022 \\
& G24 & 26.4 & 0.259 & 0.292 & 1.5 & 0.032 \\
3 & G31 & 24.3 & 0.290 & 0.280 & 0.41 & 0.028 \\
& G32 & 34.5 & 0.261 & 0.205 & 1.96 & 0.029 \\
& G33 & 20.0 & 0.10 & 0.069 & 0.0 & 0.012 \\
\hline
\end{tabular}

Table 9

Control parameters for studied AC/DC model with VSP.

\begin{tabular}{lc}
\hline Parameters & Value \\
\hline$K_{f 1, \text { vSP }}$ (p.u. MW/rad) & 1.8 \\
$K_{f 3, \text { vSP }}$ (p.u. MW/rad) & -4.5 \\
$K_{\mathrm{AC}, \text { vSP }}$ (p.u. MW) & 0.015 \\
$\omega_{n 1}(\mathrm{rad} / \mathrm{s})$ & 1.3 \\
$\omega_{n 3}(\mathrm{rad} / \mathrm{s})$ & 4.1 \\
$\zeta_{1}(\mathrm{pu})$ & 0.8 \\
$\zeta_{3}(\mathrm{pu})$ & 2.6 \\
\hline
\end{tabular}

both converters of HVDC line are equipped with VSP control to improve local and global stability of the system.

The simulation results of the three-area test system including VSP based HVDC line are also compared with the system including a normal DC and normal AC lines. In Fig. 25, the frequency deviation of all areas following the applied load disturbances in Area3 is presented. These figures show the superior performance of the proposed VSP based method to the classical model in deriving the frequency deviation close to 0 with a better dynamic.

The dynamic responses of generated power for all the generation units in different areas are depicted in Figs. 26-28. It is clear that the units in Area3, the area with 0.1 p.u. load change, are responding equally to regulate their area control error. The tieline power interchanges of all areas are shown in Fig. 29. It can be seen that the tie-line power flows in the normal systems without VSP, show more oscillations and poor performance in keeping the
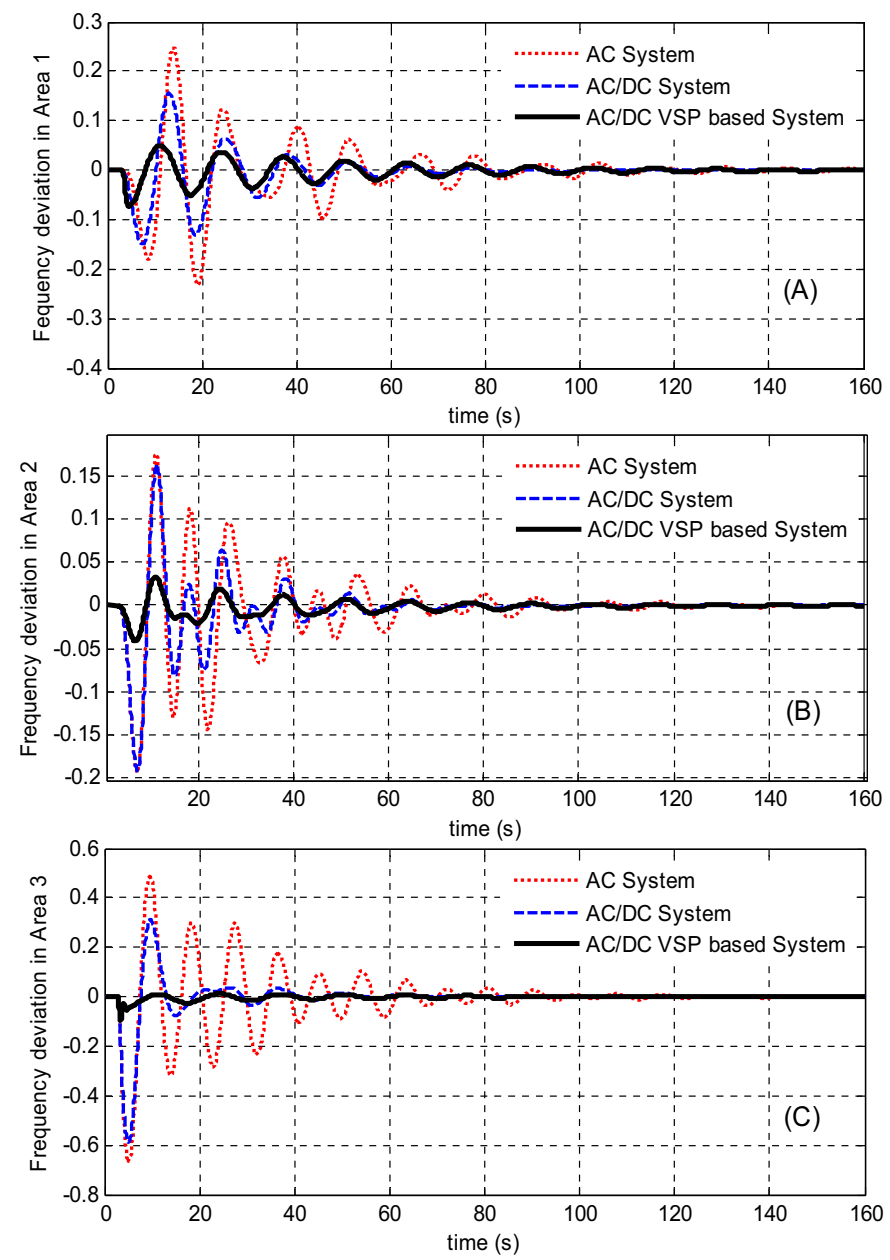

Fig. 25. Dynamic response of frequency deviations for three-area system ( $\mathrm{rad} / \mathrm{sec}$ ); (A) Area1, (B) Area2 and (C) Area3. 

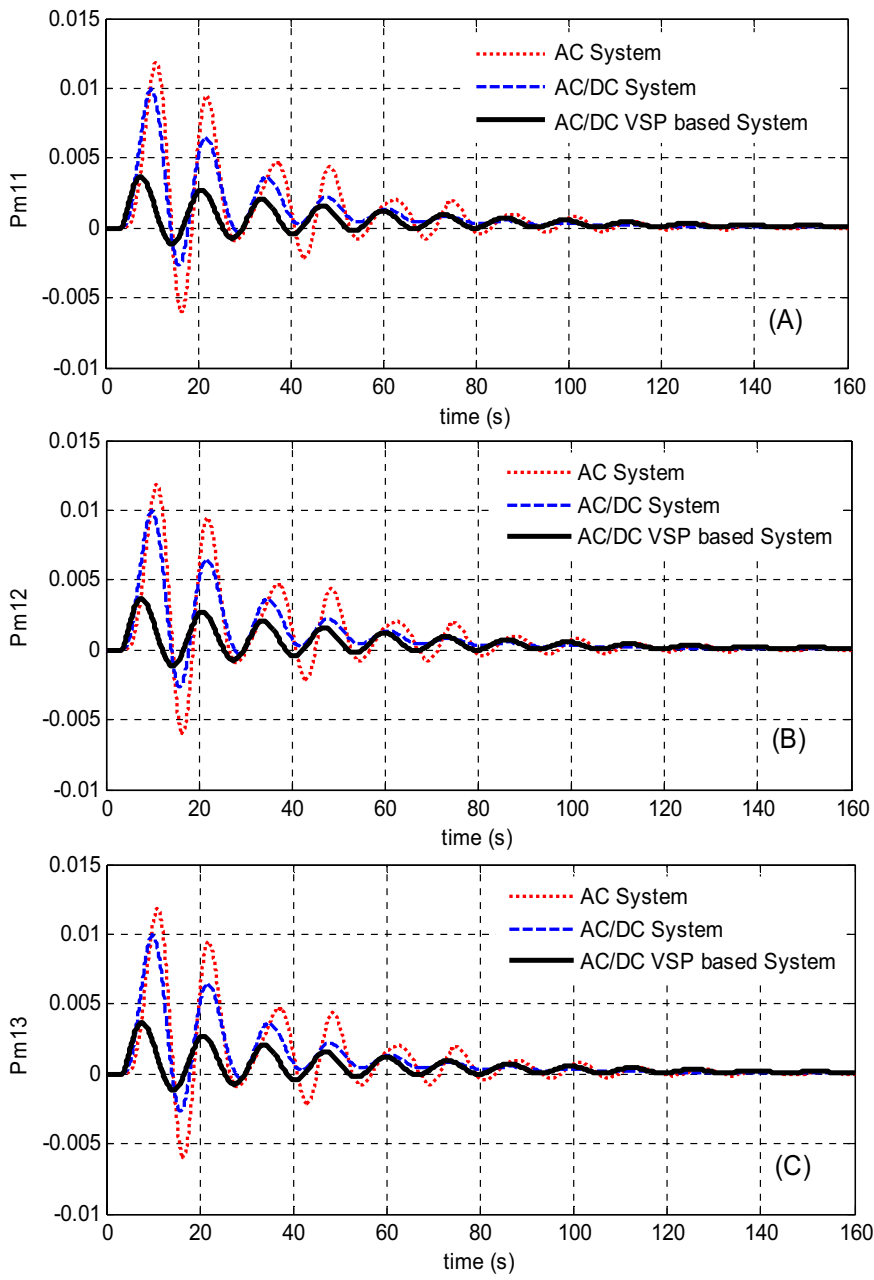

Fig. 26. Power generations in Area1; (A) GENCO11, (B) GENCO12, and (C) GENCO13.
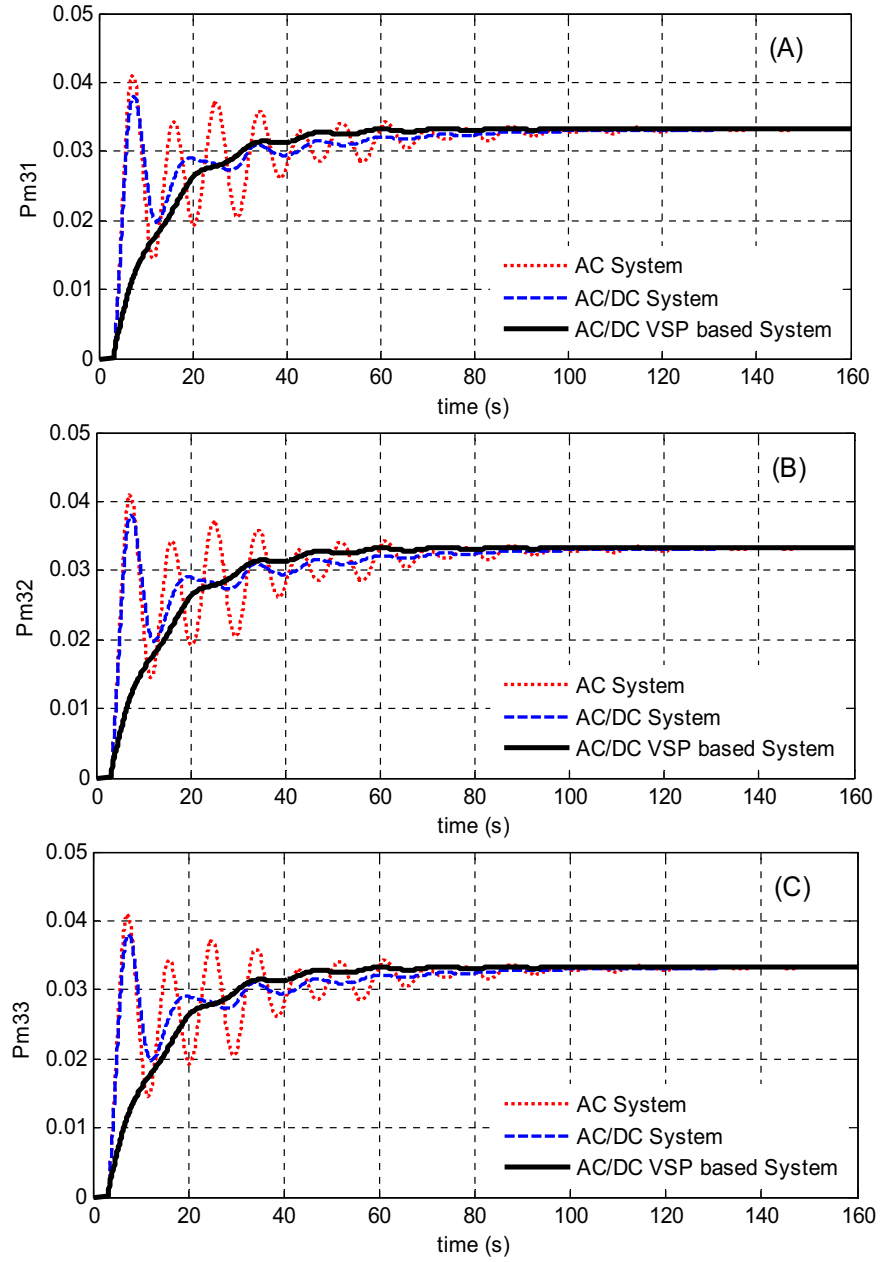

Fig. 27. Power generations in Area3; (A) GENCO31, (B) GENCO32, and (C) GENCO33.
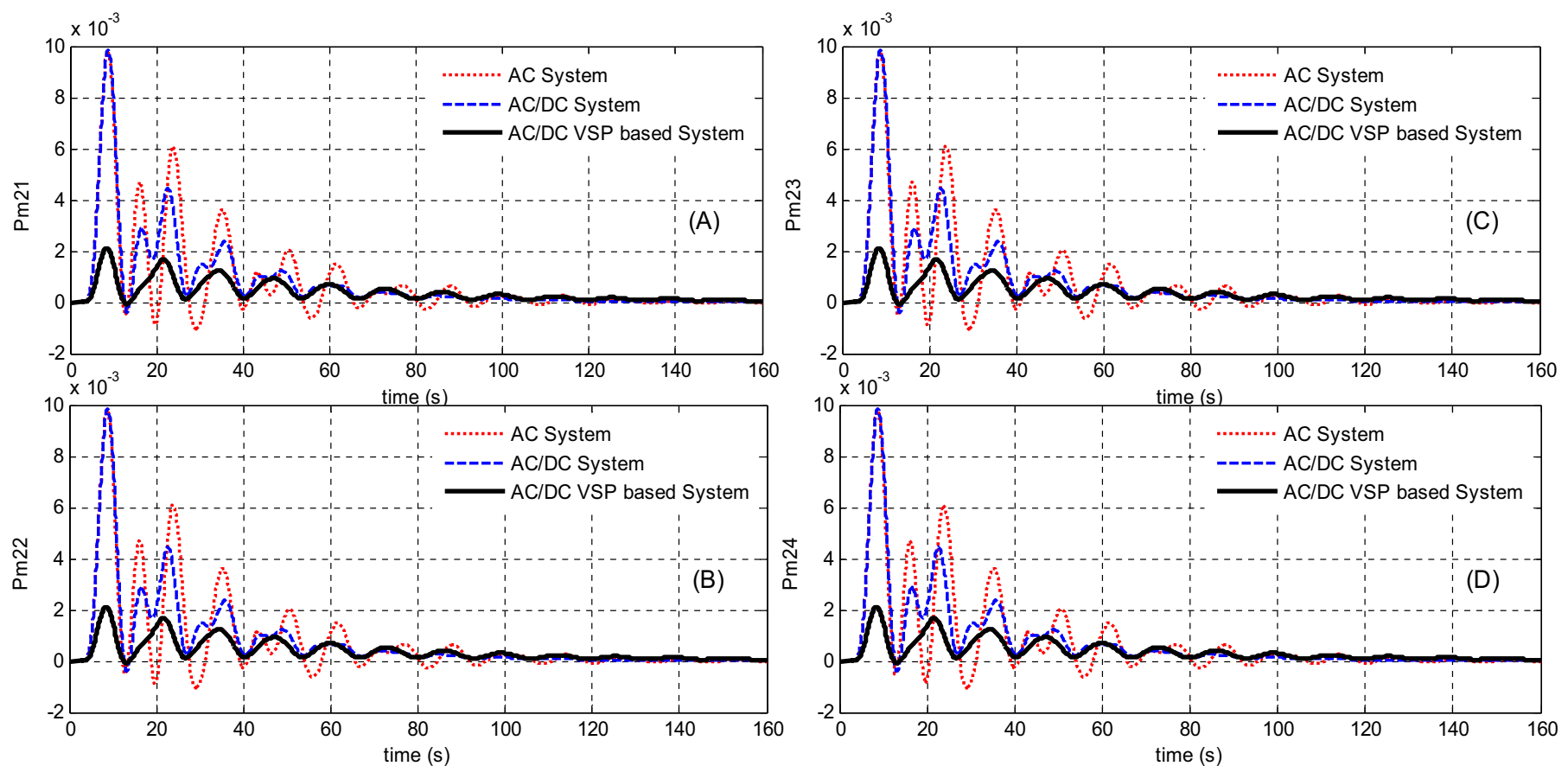

Fig. 28. Power generations in Area2; (A) GENCO21, (B) GENCO22, (C) GENCO23, and GENCO24. 

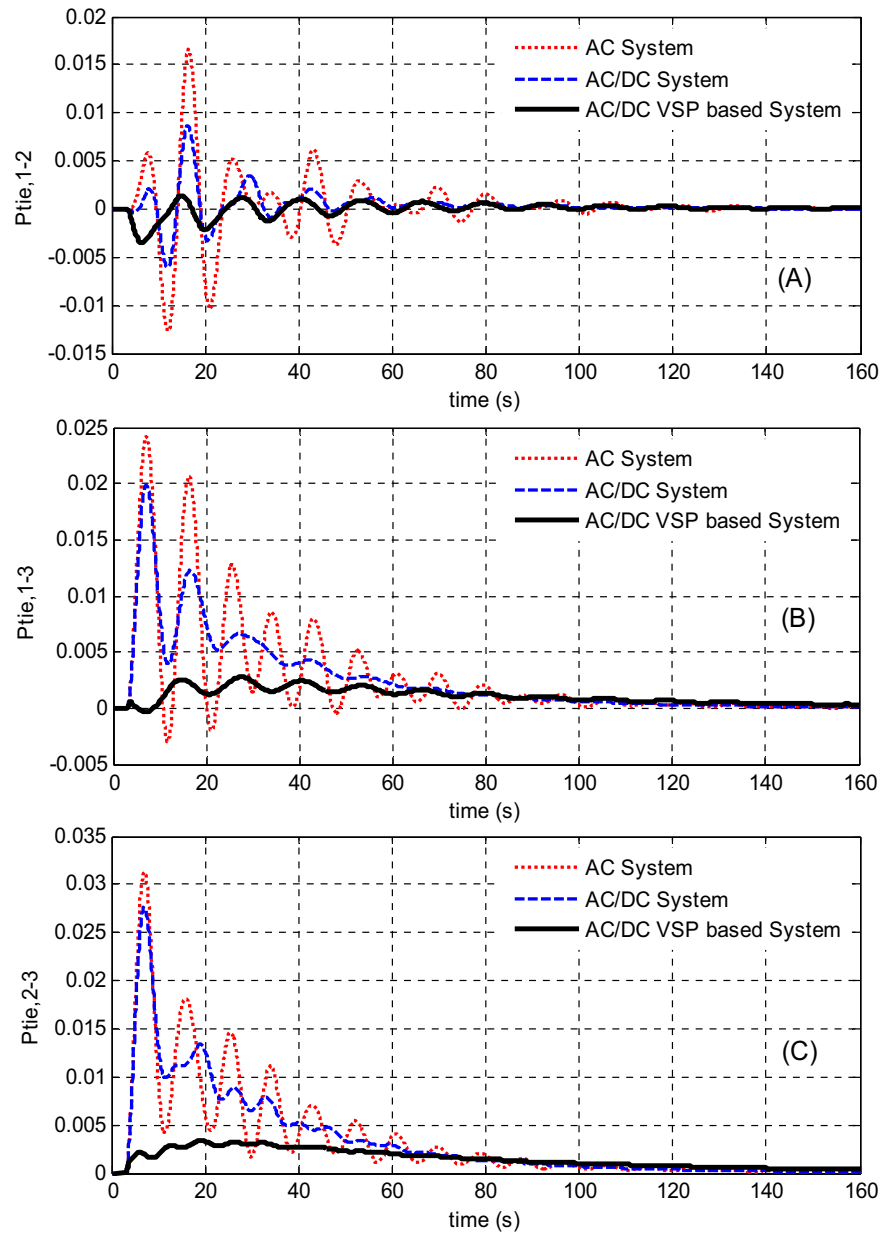

Fig. 29. Tie-line AC power exchanges; (A) Line 1-2, (A) Line 1-3, and (A) Line 2-3.

tie-line power interchanges in the scheduled values. The emulated power by VSP stations in DC link is also presented in Fig. 30. Based on the obtained result for this case, the main part of the emulated power is related to VSP3 located in Area3, the area with contingency. Based on the obtained results, the reference power for VSP system is clarified in Fig. 30. Based on each study case and considering the worst case scenario, the proper ratings of power converters with the required amount of energy can be obtained which will be helpful for pre-design stages.

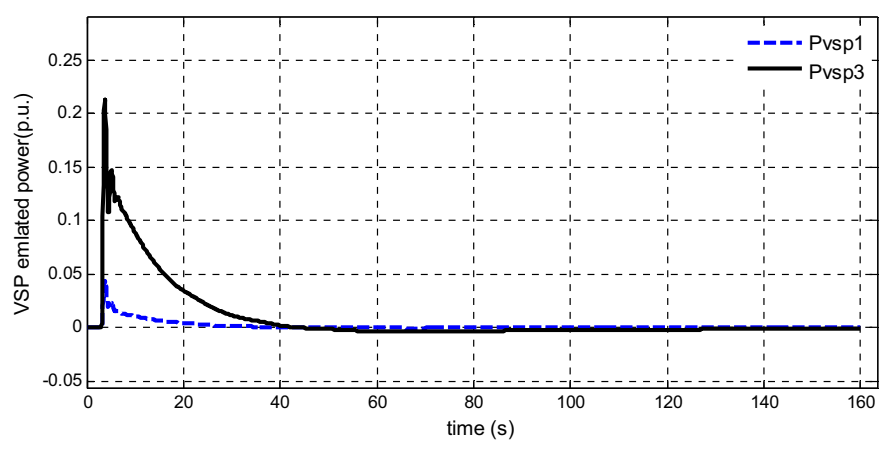

Fig. 30. Emulated power deviation form VSP based HVDC system; VSP1 in Area1 and VSP3 in Area3.

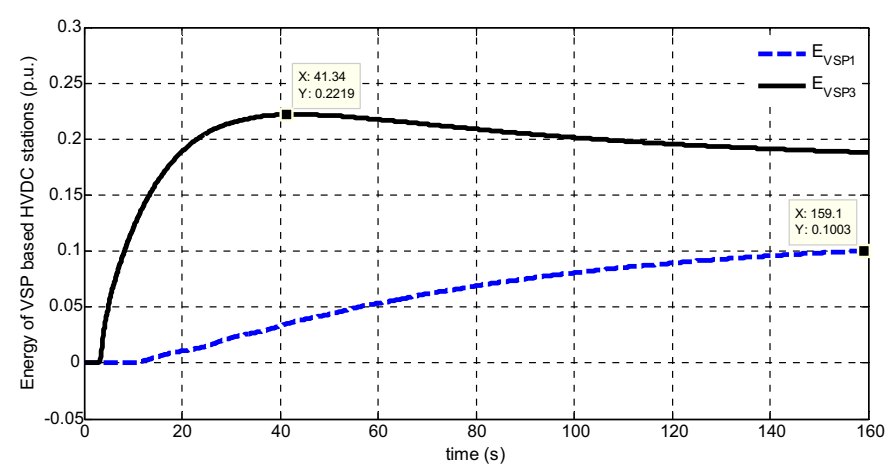

Fig. 31. Required energy by VSP based HVDC stations (p.u.).

In practice, the required energy for emulating sufficient virtual inertia in period of $t$ can be directly calculated according to the following equation for period of $t$ :

$E_{\mathrm{VSP}, i}=\int_{0}^{t} P_{\mathrm{VSP}, i}(t) \mathrm{d} t+E_{\mathrm{VSP} 0, i}$

where $P_{\mathrm{VSP}, i}$ was the instantaneous emulated power of VSP components ( $\left.\triangle X_{1, \mathrm{VSP}, i}\right)$ and $E_{\mathrm{VSP} O, i}$ is the initial energy. For this study, the energy trace is depicted by Fig. 31 in p.u. The required energy for VSP1 is 0.1 p.u. and the energy for VSP3 is equal to 0.22 p.u. Thus, knowing the base values for each application, the real values of energy with the required DC capacitance can be calculated.

\section{Conclusion}

A novel approach for modeling and analyzing the multi-area AGC scheme in the presence of VSP based inertia emulation of HVDC interconnected systems is proposed and discussed. The proposed control of VSP is a power electronics based synchronous generator which is naturally synchronized with the electrical grid by balancing the exchange of power with such grid. Therefore, it does not require any external synchronization system, such as a PLL, to work. This methodology has been proposed to cope with the future requirements of modern power system in the matter of low inertia. Proposed model has been discussed and analyzed through different eigenvalue and sensitivity analysis. The performance of the proposed approach has been also verified through different simulation for two and three area interconnected systems. Obtained results reveal that the proposed technique gives a very good dynamic performance and usages of this method will reduce the peak deviations of frequencies and tie-line power with significant improvement of the system stability.

Proposed approaches and analysis in this paper will be essential for further research in this topic. Future work will be required to explore how the inertia emulation can be implemented on the present systems with more detail, and how the VSP system can be implemented in more complex multi terminal grids. Considering different point of views of such problem like the effects of communications and SCADA system and required infrastructure for implementing such scenarios is surely a worthy area of research which will benefit the electrical industry in the future.

\section{Acknowledgments}

This work was partially supported by the Spanish Science Ministry of Economy and Competitiveness under the projects ENE2013-48428-C2-2-R and ENE2014-60228-R. Any opinions, findings and conclusions or recommendations expressed in this 
material are those of the authors and do not necessarily reflect those of the host institutions or funders.

\section{References}

[1] P. Kundur, Power System Stability and Control, McGraw-Hill, 1994.

[2] H. Saadat, Power System Analysis, McGraw-Hill, 1999.

[3] E. Rakhshani, J. Sadeh, Practical viewpoints on load frequency control problem in a deregulated power system, Energy Convers. Manage. 51 (6) (2010) 1148-1156.

[4] S.S. Koohi-Kamali, V.V. Tyagi, N.A. Rahim, N.L. Panwar, H. Mokhlis, Emergence of energy storage technologies as the solution for reliable operation of smart power systems: a review, Renew. Sustain. Energy Rev. 25 (September) (2013) $135-165$.

[5] Z.E. Al-Haiki, A.N. Shaikh-Nasser, Power transmission to distant offshore facilities, IEEE Trans. Ind. Appl. 47 (3) (2011).

[6] O.A. Giddani, A.Y.M. Abbas, G.P. Adam, O. Anaya-Lara, K.L. Lo, Multi-task control for VSC-HVDC power and frequency control, Electr. Power Energy Syst. 53 (2013) 684-690.

[7] Lingling Fan, Zhixin Miao, Dale Osborn, Wind farms with HVDC delivery in load frequency control, IEEE Trans. Power Syst. 24 (4) (2009).

[8] Du Cuiqing, E. Agneholm, G. Olsson, Use of VSC-HVDC for industrial systems having onsite generation with frequency control, IEEE Trans. Power Deliv. 23 (4) (2008) 2233-2240.

[9] Z.B. Du, Y. Zhang, L. Liu, X.H. Guan, Y.X. Ni, F.F. Wu, Structure-preserved powerfrequency slow dynamics simulation of interconnected AC/DC power systems with AGC consideration, IET Gener. Transm. Distrib. 1 (2007) 920-927.

[10] Ahmet Mete Vural, Contribution of high voltage direct current transmission systems to inter-area oscillation damping: a review, Renew. Sustain. Energy Rev. 57 (2016) 892-915.

[11] H. Bevrani, T. Hiyama, Intelligent Automatic Generation Control, CRC Press, 2011.
[12] E. Rakhshani, D. Remon, A. Mir Cantarellas, P. Rodriguez, Analysis of derivative control based virtual inertia in multi-area high-voltage direct current interconnected power systems, IET Gener. Transm. Distrib. 10 (6) (2016) 1458-1469.

[13] Jiebei Zhu, Josep M. Guerrero, Generic inertia emulation controller for multiterminal voltage-source-converter high voltage direct current systems, IET Renew. Power Gener. 8 (7) (2014) 740-748.

[14] J. Zhu, C.D. Booth, G.P. Adam, A.J. Roscoe, C.G. Bright, Inertia emulation control strategy for VSC-HVDC transmission systems, IEEE Trans. Power Syst. 28 (May (2)) (2013).

[15] P. Rodriguez, Synchronous power controller of a generation system based on static power converters, in: International Patent Application WO 2012/117131, September 7th 2012, 2012.

[16] P. Rodriguez, J. Ignacio Candela, A. Luna, Control of PV generation systems using a synchronous power controller, in: IEEE Energy Conversion Congress and Exposition, ECCE, USA, 2013.

[17] V. Donde, A. Pai, I.A. Hiskens, Simulation and optimization in an AGC system after deregulation, IEEE Trans. Power Syst. 16 (2001).

[18] M. Datta, T. Senjyu, A. Yona, T. Funabashi, K. Chul-Hwan, A frequency-control approach by photovoltaic generator in a PV-diesel hybrid power system, IEEE Trans. Energy Convers. 26 (2011) 559-571.

[19] S. Ali Pourmousavi, M. Hashem Nehrir, Introducing dynamic demand response in the LFC model, IEEE Trans. Power Syst. 29 (4) (2014) 1562-1572.

[20] E. Rakhshani, D. Remon, P. Rodriguez, Effects of PLL and frequency measurements on LFC problem in multi-area HVDC Interconnected systems, Int. J. Electr. Power Energy Syst. 81 (2016) 140-152.

[21] Yao Xu, Fangxing Li, Zhiqiang Jin, Maryam Hassani Variani, Dynamic gaintuning control (DGTC) approach for AGC with effects of wind power, IEEE Trans. Power Syst. (2016), http://dx.doi.org/10.1109/TPWRS.2015.2489562 (Early access).

[22] M.H. Variani, K. Tomsovic, Distributed automatic generation control using flatness-based approach for high penetration of wind generation, IEEE Trans. Power Syst. 28 (3) (2013).

[23] H. Bevrani, Robust Power System Frequency Control, 2nd ed., Springer, 2014. 